\title{
A dynamical point of view of Quantum Information: entropy and pressure
}

\author{
A. Baraviera, C. F. Lardizabal, A. O. Lopes, and M. Terra Cunha
}

\begin{abstract}
Quantum Information is a new area of research which has been growing rapidly since last decade. This topic is very close to potential applications to the so called Quantum Computer. In our point of view it makes sense to develop a more "dynamical point of view" of this theory. We want to consider the concepts of entropy and pressure for "stationary systems" acting on density matrices which generalize the usual ones in Ergodic Theory (in the sense of the Thermodynamic Formalism of R. Bowen, Y. Sinai and D. Ruelle). We consider the operator $\mathcal{L}$ acting on density matrices $\rho \in \mathcal{M}_{N}$ over a finite $N$-dimensional complex Hilbert space $\mathcal{L}(\rho):=\sum_{i=1}^{k} \operatorname{tr}\left(W_{i} \rho W_{i}^{*}\right) V_{i} \rho V_{i}^{*}$, where $W_{i}$ and $V_{i}, i=1,2, \ldots k$ are operators in this Hilbert space. $\mathcal{L}$ is not a linear operator. In some sense this operator is a version of an Iterated Function System (IFS). Namely, the $V_{i}(.) V_{i}^{*}=: F_{i}(),. i=1,2, \ldots, k$, play the role of the inverse branches (acting on the configuration space of density matrices $\rho$ ) and the $W_{i}$ play the role of the weights one can consider on the IFS. We suppose that for all $\rho$ we have that $\sum_{i=1}^{k} \operatorname{tr}\left(W_{i} \rho W_{i}^{*}\right)=1$. A family $W:=\left\{W_{i}\right\}_{i=1, \ldots, k}$ determines a Quantum Iterated Function System (QIFS) $\mathcal{F}_{W}, \mathcal{F}_{W}=\left\{\mathcal{M}_{N}, F_{i}, W_{i}\right\}_{i=1, \ldots, k}$.
\end{abstract}

\footnotetext{
A. T. Baraviera

I.M. - UFRGS, Porto Alegre - 91500-000, Brasil, e-mail: atbaraviera@gmail.com

C. F. Lardizabal

I.M. - UFRGS, Porto Alegre - 91500-000, Brasil, e-mail: carlos.lardizabal@gmail.com

A. O. Lopes

I.M. - UFRGS, Porto Alegre - 91500-000, Brasil, e-mail: arturoscar.lopes@gmail.com

M. Terra Cunha

D. M - UFMG, Belo Horizonte - 30161-970, Brasil, e-mail: marcelo.terra.cunha@gmail.com
} 
Dynamics, Games and Science I, DYNA 2008, Edit. M. Peixoto, A. Pinto and D Rand, pp 81-122 Springer Verlag (2011)

\section{Introduction}

We will present a survey, and also some new results, of certain topics in Quantum Information from a strictly mathematical point of view. This area is very close to potential applications to the so called Quantum Computer 26. In our point of view it makes sense to develop a more "dynamical point of view" of this theory. For instance, Von Neumann entropy is a very nice and useful concept, but, in our point of view, it is not a dynamical entropy. A nice exposition about this theory from an Ergodic Theory point of view is presented in [3] (see also [4]). Our setting is different. Part of our work is to justify why the concepts we present here are natural generalizations of the usual ones in Thermodynamic Formalism.

We have to analyze first the fundamental concepts in both theories. It is well-known that the so called Quantum Stochastic Processes have some special features which present a quite different nature than the usual classical Stochastic Processes. A main issue on QSP is the possibility of interference (see [1] 2] 8] 28, 31]). We will analyze carefully Quantum Iterated Function Systems, which were described previously by [22] and [29].

We refer the reader to 1 for the proofs of the results presented in the first part of this exposition.

Density matrices play the role of probabilities on Quantum Mechanics. In this work we investigate a generalization of the classical Thermodynamic Formalism (in the sense of Bowen, Sinai and Ruelle) for the setting of density matrices. We consider the operator $\mathcal{L}$ acting on density matrices $\rho \in \mathcal{M}_{N}$ over a finite $N$-dimensional complex Hilbert space

$$
\mathcal{L}(\rho):=\sum_{i=1}^{k} \operatorname{tr}\left(W_{i} \rho W_{i}^{*}\right) V_{i} \rho V_{i}^{*},
$$

where $W_{i}$ and $V_{i}, i=1,2, . . k$ are operators in this Hilbert space. Note that $\mathcal{L}$ is not a linear operator.

In some sense this operator is a version of an Iterated Function System (IFS). Namely, the $V_{i}(.) V_{i}^{*}=: F_{i}(),. i=1,2, \ldots, k$, play the role of the inverse branches (acting on the configuration space of density matrices $\rho$ ) and the $W_{i}$ play the role of the weights one can consider on the IFS. We suppose that for all $\rho$ we have that $\sum_{i=1}^{k} \operatorname{tr}\left(W_{i} \rho W_{i}^{*}\right)=1$. This means that $\mathcal{L}_{\mathcal{F}_{W}}$ is a normalized operator. 
A family $W:=\left\{W_{i}\right\}_{i=1, \ldots, k}$ determines a Quantum Iterated Function System (QIFS) $\mathcal{F}_{W}$,

$$
\mathcal{F}_{W}=\left\{\mathcal{M}_{N}, F_{i}, W_{i}\right\}_{i=1, \ldots, k}
$$

We want to consider a new concept of entropy for stationary systems acting on density matrices which generalizes the usual one in Ergodic Theory. In our setting the $V_{i}, i=1,2, \ldots, k$ are fixed (i.e. the dynamics of the inverse branches is fixed in the beginning) and we consider the different families $W_{i}$, $i=1,2, \ldots, k$, (also with the attached corresponding eigendensity matrix $\rho_{W}$ ) as possible Jacobians (of "stationary probabilities").

It is appropriate to make here a remark about the meaning of "stationarity" for us. In Ergodic Theory the action of the shift $\sigma$ in the Bernoulli space $\Omega=\{1,2, \ldots, k\}^{\mathbb{N}}$ with $k$ symbols is well understood. The concept of stationarity for a Stochastic Process (where the space of states is $S=\{1,2, \ldots, k\}$ ) is defined by the shift-invariance for the associated probability $P$ in the Bernoulli space (the space of paths). Shannon-Kolmogorov entropy is a concept designed for stationary probabilities. When the probability $P$ is associated to a Markov chain, this entropy is given by

$$
H(P):=-\sum_{i, j=1}^{N} p_{i} p_{i j} \log p_{i j}
$$

where $P=\left(p_{i j}\right)$ describes the transition matrix, and $p_{i}$ the invariant probability vector, $i, j=1,2, . ., k$. This is the key idea for our definition of stationary entropy.

Thermodynamic Formalism and the Ruelle operator for a potential $A$ : $\Omega \rightarrow \mathbb{R}$ are natural generalizations of the theory associated to the Perron theorem for positive matrices (see [30]) (this occurs when the potential depends on only the first two symbols of $\left.w=\left(w_{1}, w_{2}, w_{3}, \ldots\right) \in \Omega\right)$. We will analyze the Pressure problem for density matrices under this last perspective.

The main point here (and also in [1] 2, 18, 20]) is that in order to define Kolmogorov entropy one can avoid the use of partitions, etc. We just need to look the problem at the level of Ruelle operators (which in some sense captures the underlying dynamics).

Given a normalized family $W_{i}, i=1,2, . ., k$, a natural definition of entropy, denoted by $h_{V}(W)$, is given by

$$
-\sum_{i=1}^{k} \frac{\operatorname{tr}\left(W_{i} \rho_{W} W_{i}^{*}\right)}{\operatorname{tr}\left(V_{i} \rho_{W} V_{i}^{*}\right)} \sum_{j=1}^{k} \operatorname{tr}\left(W_{j} V_{i} \rho_{W} V_{i}^{*} W_{j}^{*}\right) \log \left(\frac{\operatorname{tr}\left(W_{j} V_{i} \rho_{W} V_{i}^{*} W_{j}^{*}\right)}{\operatorname{tr}\left(V_{i} \rho_{W} V_{i}^{*}\right)}\right),
$$


where, $\rho_{W}$ denotes the barycenter of the unique invariant, attractive measure for the Markov operator $\mathcal{V}$ associated to $\mathcal{F}_{W}$. We show that this generalizes the entropy of a Markov System. This will be described later on this work.

A different definition of entropy for density operators is presented in 2 [7. There are examples where the values one gets from these two concepts are different (see [2]).

We also want to present here a concept of pressure for stationary systems acting on density matrices which generalizes the usual one in Ergodic Theory.

In addition to the dynamics obtained by the $V_{i}$, which are fixed, a family of potentials $H_{i}, i=1,2, \ldots k$ induces a kind of Ruelle operator given by

$$
\mathcal{L}_{H}(\rho):=\sum_{i=1}^{k} \operatorname{tr}\left(H_{i} \rho H_{i}^{*}\right) V_{i} \rho V_{i}^{*}
$$

We show that such operator admits an eigenvalue $\beta$ and an associated eigenstate $\rho_{\beta}$, that is, one satisfying $\mathcal{L}_{H}\left(\rho_{\beta}\right)=\beta \rho_{\beta}$.

The natural generalization of the concept of pressure for a family $H_{i}$, $i=1,2, \ldots k$ is the problem of finding the maximization on the possible normalized families $W_{i}, i=1,2, \ldots k$, of the expression

$$
h_{V}(W)+\sum_{j=1}^{k} \log \left(\operatorname{tr}\left(H_{j} \rho_{H} H_{j}^{*}\right) \operatorname{tr}\left(V_{j} \rho_{H} V_{j}^{*}\right)\right) \operatorname{tr}\left(W_{j} \rho_{W} W_{j}^{*}\right)
$$

We show a relation between the eigendensity matrix $\rho_{H}$ for the Ruelle operator and the set of $W_{i}, i=1,2, \ldots k$, which maximizes pressure. In the case each $V_{i}, i=1,2, \ldots k$, is unitary, then the maximum value is $\log \beta$.

Our work is inspired by the results presented in 22 and 29 . We would like to thank these authors for supplying us with the corresponding references.

We point out that completely positive mappings (operators) acting on density matrices are of great importance in Quantum Computing. These operators can be written in the Stinespring-Kraus form. This motivates the study of operators in the class we will assume here, which are a generalization of such Stinespring-Kraus transformations.

The initial part of our work is dedicated to present all the definitions and concepts that are not well-known (at least for the general audience of people in Dynamical Systems), in a systematic and well organized way. We present many examples and all the basic main definitions which are necessary to understand the theory. However, we do not have the intention to exhaust what is already known. We believe that the theoretical results presented here can be useful as a general tool to understand problems in Quantum Computing. 
Several examples are presented with all details in the text. We believe that this will help the reader to understand the main issues of the theory.

In order to simplify the notation we will present most of our results for the case of two by two matrices.

In sections 2 and 3 we present some basic definitions, examples and we show some preliminary relations of our setting to the classical Thermodynamic Formalism. In section 4 we present an eigenvalue problem for nonnormalized Ruelle operators which will be required later. Some properties and concepts about density matrices and Ruelle operators are presented in sections 6 and 7. In section 10 we introduce the concept of stationary entropy for measures defined on the set of density matrices. In section 11 we compare this definition with the usual one for Markov Chains. Section 12 aims to motivate the interest on pressure and the capacity-cost function. The sections [13 ??, 14 and 15 are dedicated to the presentation of our main results on pressure, important inequalities, examples and its relation with the classical theory of Thermodynamic Formalism.

This work is part of the thesis dissertation of C. F. Lardizabal in Prog. Pos-Grad. Mat. UFRGS (Brazil) [16].

\section{Basic definitions}

Let $M_{N}(\mathbb{C})$ the set of complex matrices of order $N$. If $\rho \in M_{N}(\mathbb{C})$ then $\rho^{*}$ denotes the transpose conjugate of $\rho$. We consider in $\mathbb{C}^{N}$ the $\mathcal{L}^{2}$ norm. A state (or vector) in $\mathbb{C}^{N}$ will be denoted by $\psi$ or $|\psi\rangle$, and the associated projection will be written $|\psi\rangle\langle\psi|$. Define

$$
\begin{gathered}
\mathcal{H}_{N}:=\left\{\rho \in M_{N}(\mathbb{C}): \rho^{*}=\rho\right\} \\
\mathcal{P H}_{N}:=\left\{\rho \in \mathcal{H}_{N}:\langle\rho \psi, \psi\rangle \geq 0, \forall \psi \in \mathbb{C}^{N}\right\} \\
\mathcal{M}_{N}:=\left\{\rho \in \mathcal{P} \mathcal{H}_{N}: \operatorname{tr}(\rho)=1\right\} \\
\mathcal{P}_{N}:=\left\{\rho \in \mathcal{H}_{N}: \rho=|\psi\rangle\langle\psi|, \psi \in \mathbb{C}^{N},\langle\psi \mid \psi\rangle=1\right\},
\end{gathered}
$$

the space of hermitian, positive, density operators and pure states, respectively. Density operators are also called mixed states. Any state $\rho$, by the spectral theorem, can be written as

$$
\rho=\sum_{i=1}^{k} p_{i}\left|\psi_{i}\right\rangle\left\langle\psi_{i}\right|,
$$


for some choice of $p_{i}$, which are positive numbers with $\sum_{i} p_{i}=1$, and $\psi_{i}$, which have norm one and are orthogonal.

The set $\mathcal{P}_{N}$ is the set of extremal points of $\mathcal{M}_{N}$, that is, the set of points which can not be decomposed as a nontrivial convex combination of elements in $\mathcal{M}_{N}$.

Definition 1 Let $G_{i}: \mathcal{M}_{N} \rightarrow \mathcal{M}_{N}, p_{i}: \mathcal{M}_{N} \rightarrow[0,1], i=1, \ldots, k$ and such that $\sum_{i} p_{i}(\rho)=1$. We call

$$
\mathcal{F}_{N}=\left\{\mathcal{M}_{N}, G_{i}, p_{i}: i=1, \ldots, k\right\}
$$

$a$ Quantum Iterated Function System (QIFS).

Definition $2 A$ QIFS is homogeneous if $p_{i}$ and $G_{i} p_{i}$ are affine mappings, $i=1, \ldots, k$.

Suppose that the QIFS considered is such that there are $V_{i}$ and $W_{i}$ linear maps, $i=1, \ldots, k$, with $\sum_{i=1}^{k} W_{i}^{*} W_{i}=I$ such that

$$
G_{i}(\rho)=\frac{V_{i} \rho V_{i}^{*}}{\operatorname{tr}\left(V_{i} \rho V_{i}^{*}\right)}
$$

and

$$
p_{i}(\rho)=\operatorname{tr}\left(W_{i} \rho W_{i}^{*}\right)
$$

Then we have that a QIFS is homogeneous if $V_{i}=W_{i}, i=1, \ldots, k$.

Now we can define a Markov operator $\mathcal{V}: \mathcal{M}\left(\mathcal{M}_{N}\right) \rightarrow \mathcal{M}\left(\mathcal{M}_{N}\right)$,

$$
(\mathcal{V} \mu)(B)=\sum_{i=1}^{k} \int_{G_{i}^{-1}(B)} p_{i}(\rho) d \mu(\rho),
$$

where $\mathcal{M}\left(\mathcal{M}_{N}\right)$ denotes the space of probability measure over $\mathcal{M}_{N}$. We also define $\Lambda: \mathcal{M}_{N} \rightarrow \mathcal{M}_{N}$,

$$
\Lambda(\rho):=\sum_{i=1}^{k} p_{i}(\rho) G_{i}(\rho)
$$

The operator defined above has no counterpart in the classical Thermodynamic Formalism. We will also consider the operator acting on density matrices $\rho$.

$$
\mathcal{L}(\rho)=\sum_{i=k}^{k} q_{i}(\rho) V_{i} \rho V_{i}^{*} .
$$

If for all $\rho$ we have $\sum_{i=k}^{k} q_{i}(\rho)=1$, we say the operator is normalized. 
In the normalized case, the different possible choices of $q_{i}, i=1,2, \ldots, k$, (which means different choices of $W_{i}, i=1,2, \ldots, k$ ) play here the role of the different Jacobians of possible invariant probabilities (see 23. II. 1, and [20]) in Thermodynamic Formalism. In some sense the probabilites can be identified with the Jacobians (this is true at least for Gibbs probabilities of Hölder potentials [25]). The set of Gibbs probabilities for Hölder potentials is dense in the set of invariant probabilities [19.

We are also interested on the non-normalized case. If the QIFS is homogeneous, then

$$
\Lambda(\rho)=\sum_{i} V_{i} \rho V_{i}^{*}
$$

Theorem 1 [29] A mixed state $\rho_{0}$ is $\Lambda$-invariant if and only if

$$
\rho_{0}=\int_{\mathcal{M}_{N}} \rho d \mu(\rho)
$$

for some $\mathcal{V}$-invariant measure $\mu$.

In order to define hyperbolic QIFS, one has to define a distance on the space of mixed states. For instance, we could choose one of the following:

$$
\begin{gathered}
D\left(\rho_{1}, \rho_{2}\right)=\sqrt{\operatorname{tr}\left[\left(\rho_{1}-\rho_{2}\right)^{2}\right]} \\
D\left(\rho_{1}, \rho_{2}\right)=\operatorname{tr} \sqrt{\left(\rho_{1}-\rho_{2}\right)^{2}} \\
D\left(\rho_{1}, \rho_{2}\right)=\sqrt{2\left\{1-\operatorname{tr}\left[\left(\rho_{1}^{1 / 2} \rho_{2} \rho_{1}^{1 / 2}\right)^{1 / 2}\right]\right\}}
\end{gathered}
$$

Such metrics generate the same topology on $\mathcal{M}$. Considering the space of mixed states with one of those metrics we can make the following definition. We say that a QIFS is hyperbolic if the quantum maps $G_{i}$ are contractions with respect to one of the distances on $\mathcal{M}_{N}$ and if the maps $p_{i}$ are Höldercontinuous and positive, see for instance, 22.

Proposition 1 If a QIFS (3) is homogeneous and hyperbolic the associated Markov operator admits a unique invariant measure $\mu$. Such invariant measure determines a unique $\Lambda$-invariant state $\rho \in \mathcal{M}_{N}$, given by (7).

See 22, 29] for the proof. 


\section{Examples of QIFS}

Example $1 \Omega=\mathcal{M}_{N}, k=2, p_{1}=p_{2}=1 / 2, G_{1}(\rho)=U_{1} \rho U_{1}^{*}, G_{2}(\rho)=$ $U_{2} \rho U_{2}^{*}$. The normalized identity matrix $\rho_{*}=I / N$ is $\Lambda$-invariant, for any choice of unitary $U_{1}$ and $U_{2}$. Note that we can write

$$
\rho_{*}=\int_{\mathcal{M}_{N}} \rho d \mu(\rho)
$$

where the measure $\mu$, uniformly distributed over $\mathcal{P}_{N}$, is $\mathcal{V}$-invariant.

In the example described below we use Dirac notation for the projections.

Example 2 We are interested in finding the fixed point $\hat{\rho}$ for $\Lambda$ in an example for the case $N=2$ and $k=3$.

Consider the bits $\mid 0>=(0,1)$ and $\mid 1>=(1,0)$ (the canonical basis). The states $\rho$ are generated by $|0><0|,|0><1|,|1><0|$ and $|1><1|$. Take $V_{1}=I$ and $V_{2}$ such that $|0>\rightarrow| 0>$ and $|1>\rightarrow| 0>$. Consider $V_{3}$ such that $|0>\rightarrow| 1>$ and $|1>\rightarrow| 1>$. That is, $V_{2}=|0><0|+|0><1|$ and $V_{3}=|1><0|+|1><1|$. Therefore, $V_{2}^{*}=|0><0|+|1><0|$ and $V_{3}^{*}=|0><1|+|1><1|$. Suppose $p_{i}=\hat{p}_{i}, i=1,2,3$, are such that $\sum_{i} p_{i}=1$ (in this case, each $p_{i}$ is independent of $\rho$ ). Therefore, we consider the operator $\mathcal{L}$ and look for fixed points $\rho$. Suppose

$$
\rho=\rho_{00}|0><0|+\rho_{01}|0><1|+\rho_{10}|1><0|+\rho_{11}|1><1|
$$

Then

$$
\begin{gathered}
\Lambda(\rho)=\sum_{i=1}^{3} p_{i}(\rho) \frac{\left(V_{i} \rho V_{i}^{*}\right)}{\operatorname{tr}\left(V_{i} \rho V_{i}^{*}\right)}= \\
\sum_{i=1}^{3} p_{i}\left[\frac{V_{i}\left(\left(\rho_{00}|0><0|+\rho_{01}|0><1|+\rho_{10}|1><0|+\rho_{11}|1><1|\right)\right) V_{i}^{*}}{\operatorname{tr}\left(V_{i} \rho V_{i}^{*}\right)}\right]
\end{gathered}
$$

Let us compute first the action of the operator $V_{2}|0><0| V_{2}^{*}$.

Note that $\left(V_{2}|0><0| V_{2}^{*}\right)\left|0>=V_{2}\right| 0><0\left|(|0>+| 1>)=V_{2}\right| 0>=$ $\mid 0>$ and $\left(V_{2}|0><0| V_{2}^{*}\right) \mid 1>=V_{2}(0)=0$. More generally

$$
\begin{gathered}
\rho V_{2}^{*}=\left(\rho_{00}|0><0|+\right. \\
\left.\rho_{01}|0><1|+\rho_{10}|1><0|+\rho_{11}|1><1|\right)(|0><0|+|1><0|)= \\
\rho_{00}|0><0|+\rho_{01}|0><0|+\rho_{10}|1><0|+\rho_{11}|1><0| .
\end{gathered}
$$

Therefore, 


$$
\begin{gathered}
V_{2} \rho V_{2}^{*}=(|0><0|+|0><1|)\left(\rho_{00}|0><0|+\right. \\
\left.\rho_{01}|0><0|+\rho_{10}|1><0|+\rho_{11}|1><0|\right)= \\
\left(\rho_{00}+\rho_{01}+\rho_{10}+\rho_{11}\right)|0><0|=\left(1+2 \operatorname{Re}\left(\rho_{01}\right)\right)|0><0|,
\end{gathered}
$$

because $\rho$ has trace $1=\rho_{00}+\rho_{11}$. Note that $\operatorname{tr}\left(V_{2} \rho V_{2}^{*}\right)=\left(1+2 \operatorname{Re}\left(\rho_{01}\right)\right)$. A similar result can be obtained for $V_{3}$. Proceeding in the same way we get that

$$
\begin{gathered}
\Lambda(\rho)=p_{1}\left(\rho_{00}|0><0|+\rho_{01}|0><1|+\rho_{10}|1><0|+\rho_{11}|1><1|\right)+ \\
p_{2}|0><0|+p_{3}|1><1| .
\end{gathered}
$$

The equation

$$
\Lambda(\rho)=\rho=\rho_{00}|0><0|+\rho_{01}|0><1|+\rho_{10}|1><0|+\rho_{11}|1><1|
$$

means

$$
\begin{gathered}
p_{1} \rho_{00}+p_{2}=\rho_{00}, \\
p_{1} \rho_{01}=\rho_{01}, \\
p_{1} \rho_{10}=\rho_{10}, \\
p_{1} \rho_{11}+p_{3}=\rho_{11} .
\end{gathered}
$$

If $p_{1} \neq 0$, then $\rho_{01}=\rho_{10}=0$. Finally, if $p_{1} \neq 1$, then $\rho_{00}=\frac{p_{2}}{1-p_{1}}$ and $\rho_{11}=\frac{p_{3}}{1-p_{1}}$ and the fixed point is

$$
\hat{\rho}=\frac{p_{2}}{1-p_{1}}|0><0|+\frac{p_{3}}{1-p_{1}}|1><1|
$$

We recall that a mapping $\Lambda$ is completely positive (CP) if $\Lambda \otimes I$ is positive for any extension of the Hilbert space considered $\mathcal{H}_{N} \rightarrow \mathcal{H}_{N} \otimes \mathcal{H}_{E}$. We know that every CP mapping which is trace-preserving can be represented (in a nonunique way) in the Stinespring-Kraus form

$$
\Lambda_{K}(\rho)=\sum_{j=1}^{k} V_{j} \rho V_{j}^{*}, \quad \sum_{j=1}^{k} V_{j}^{*} V_{j}=1,
$$

where the $V_{i}$ are linear operators. Moreover if we have $\sum_{j=1}^{k} V_{j} V_{j}^{*}=I$, then $\Lambda(I / N)=I / N$. This is the case if each of the $V_{i}$ are normal.

We call a unitary trace-preserving CP map a bistochastic map. An example of such a mapping is 


$$
\Lambda_{U}(\rho)=\sum_{i=1}^{k} p_{i} U_{i} \rho U_{i}^{*},
$$

where the $U_{i}$ are unitary operators and $\sum_{i} p_{i}=1$. Note that if we write $G_{i}(\rho)=U_{i} \rho U_{i}^{*}$, then example 1 is part of this class of operators. For such operators we have that $\rho_{*}$ is an invariant state for $\Lambda_{U}$ and also that $\delta_{\rho_{*}}$ is invariant for the Markov operator $P_{U}$ induced by this QIFS.

We will present a simple example of the kind of problems we are interested here, namely eigenvalues and eigendensity matrices. Let $\mathcal{H}_{N}$ be a Hilbert space of dimension $N$. As before, let $\mathcal{M}_{N}$ be the space of density operators on $\mathcal{H}_{N}$. A natural problem is to find fixed points for $\Lambda: \mathcal{M}_{N} \rightarrow \mathcal{M}_{N}$,

$$
\Lambda(\rho)=\sum_{i=1}^{k} V_{i} \rho V_{i}^{*}
$$

In order to simplify our reasoning we fix $N=2$ and $k=2$. Let

$$
V_{1}=\left(\begin{array}{ll}
v_{1} & v_{2} \\
v_{3} & v_{4}
\end{array}\right), \quad V_{2}=\left(\begin{array}{ll}
w_{1} & w_{2} \\
w_{3} & w_{4}
\end{array}\right), \quad \rho=\left(\begin{array}{ll}
\rho_{1} & \rho_{2} \\
\overline{\rho_{2}} & \rho_{4}
\end{array}\right)
$$

where $V_{1}$ and $V_{2}$ are invertible and $\rho$ is a density operator. We would like to find $\rho$ such that

$$
V_{1} \rho V_{1}^{*}+V_{2} \rho V_{2}^{*}=\rho .
$$

Below we have an example where the matrices $V_{i}$ are not real.

Example 3 Let

$$
V_{1}=e^{i k}\left(\begin{array}{cc}
\sqrt{p} & 0 \\
0 & -\sqrt{p}
\end{array}\right), \quad V_{2}=e^{i l}\left(\begin{array}{cc}
\sqrt{1-p} & 0 \\
0 & -\sqrt{1-p}
\end{array}\right),
$$

where $k, l \in \mathbb{R}, p \in(0,1)$. Then $V_{1}^{*} V_{1}+V_{2}^{*} V_{2}=I$. A simple calculation shows that $\rho_{2}=0$, and then

$$
\rho=\left(\begin{array}{cc}
q & 0 \\
0 & 1-q
\end{array}\right)
$$

is invariant to $\Lambda(\rho)=V_{1} \rho V_{1}^{*}+V_{2} \rho V_{2}^{*}$, for $q \in(0,1)$.

Now we make a few considerations about the Ruelle operator $\mathcal{L}$ defined before. In particular, we show that Perron's classic eigenvalue problem is a particular case of the problem for the operator $\mathcal{L}$ acting on matrices. Let 


$$
\begin{gathered}
V_{1}=\left(\begin{array}{cc}
p_{00} & 0 \\
0 & 0
\end{array}\right), \quad V_{2}=\left(\begin{array}{cc}
0 & p_{01} \\
0 & 0
\end{array}\right) \\
V_{3}=\left(\begin{array}{cc}
0 & 0 \\
p_{10} & 0
\end{array}\right), \quad V_{4}=\left(\begin{array}{cc}
0 & 0 \\
0 & p_{11}
\end{array}\right), \quad \rho=\left(\begin{array}{cc}
\rho_{1} & \rho_{2} \\
\rho_{3} & \rho_{4}
\end{array}\right)
\end{gathered}
$$

Define

$$
\mathcal{L}(\rho)=\sum_{i=1}^{4} q_{i}(\rho) V_{i} \rho V_{i}^{*}
$$

We have that $\mathcal{L}(\rho)=\rho$ implies $\rho_{2}=0$ and

$$
\begin{aligned}
& a \rho_{1}+b \rho_{4}=\rho_{1} \\
& c \rho_{1}+d \rho_{4}=\rho_{4}
\end{aligned}
$$

where

$$
a=q_{1} p_{00}^{2}, \quad b=q_{2} p_{01}^{2}, \quad c=q_{3} p_{10}^{2}, \quad d=q_{4} p_{11}^{2}
$$

Solving (9) and (10) in terms of $\rho_{1}$ gives

$$
\rho_{1}=\frac{b}{1-a} \rho_{4}, \quad \rho_{1}=\frac{1-d}{c} \rho_{4}
$$

that is,

$$
\frac{b}{1-a}=\frac{1-d}{c}
$$

which is a restriction over the $q_{i}$. For simplicity we assume here that the $q_{i}$ are constant. One can show that

$$
\rho=\left(\begin{array}{cc}
\frac{q_{2} p_{01}^{2}}{q_{2} p_{01}^{2}-q_{1} p_{00}^{2}+1} & 0 \\
0 & \frac{1-q_{1} p_{00}^{2}}{q_{2} p_{01}^{2}-q_{1} p_{00}^{2}+1}
\end{array}\right)=\left(\begin{array}{cc}
\frac{1-q_{4} p_{11}^{2}}{1-q_{4} p_{11}^{2}+q_{3} p_{10}^{2}} & 0 \\
0 & \frac{q_{3} p_{10}^{2}}{1-q_{4} p_{11}^{2}+q_{3} p_{10}^{2}}
\end{array}\right)
$$

Now let

$$
P=\sum_{i} V_{i}=\left(\begin{array}{ll}
p_{00} & p_{01} \\
p_{10} & p_{11}
\end{array}\right),
$$

be a column-stochastic matrix. Let $\pi=\left(\pi_{1}, \pi_{2}\right)$ such that $P \pi=\pi$. Then

$$
\pi=\left(\frac{p_{01}}{p_{01}-p_{00}+1}, \frac{1-p_{00}}{p_{01}-p_{00}+1}\right)
$$

Comparing (13) and (12) suggests that we should fix

$$
q_{1}=\frac{1}{p_{00}}, \quad q_{2}=\frac{1}{p_{01}}, \quad q_{3}=\frac{1}{p_{10}}, \quad q_{4}=\frac{1}{p_{11}}
$$


Then the nonzero entries of $\rho$ are equal to the entries of $\pi$ and therefore we associate the fixed point of $P$ to the fixed point of some $\mathcal{L}$ in a natural way. But note that such a choice of $q_{i}$ is not unique, because

$$
q_{2}=\frac{1-q_{1} p_{00}^{2}}{p_{01} p_{10}}, \quad q_{4}=\frac{1-q_{3} p_{10} p_{01}}{p_{11}^{2}},
$$

for any $q_{1}, q_{3}$ also produces $\rho$ with nonzero coordinates equal to the coordinates of $\pi$.

Now we consider the following problem. Let

$$
\begin{gathered}
V_{1}=\left(\begin{array}{cc}
h_{00} & 0 \\
0 & 0
\end{array}\right), \quad V_{2}=\left(\begin{array}{cc}
0 & h_{01} \\
0 & 0
\end{array}\right), \quad V_{3}=\left(\begin{array}{cc}
0 & 0 \\
h_{10} & 0
\end{array}\right) \\
V_{4}=\left(\begin{array}{cc}
0 & 0 \\
0 & h_{11}
\end{array}\right), \quad H=\sum_{i} V_{i}, \quad \rho=\left(\begin{array}{ll}
\rho_{1} & \rho_{2} \\
\rho_{3} & \rho_{4}
\end{array}\right)
\end{gathered}
$$

Define

$$
\mathcal{L}(\rho)=\sum_{i=1}^{4} q_{i} V_{i} \rho V_{i}^{*}
$$

where $q_{i} \in \mathbb{R}$. Assume that $h_{i j} \in \mathbb{R}$, so we want to obtain $\lambda$ such that $\mathcal{L}(\rho)=\lambda \rho, \lambda \neq 0$, and $\lambda$ is the largest eigenvalue. With a few calculations we obtain $\rho_{2}=\rho_{3}=0$,

$$
\begin{aligned}
& q_{1} h_{00}^{2} \rho_{1}+q_{2} h_{01}^{2} \rho_{4}=\lambda \rho_{1} \\
& q_{3} h_{10}^{2} \rho_{1}+q_{4} h_{11}^{2} \rho_{4}=\lambda \rho_{4}
\end{aligned}
$$

that is,

$$
\begin{gathered}
a \rho_{1}+b \rho_{4}=\lambda \rho_{1} \\
c \rho_{1}+d \rho_{4}=\lambda \rho_{4},
\end{gathered}
$$

with

$$
a=q_{1} h_{00}^{2}, \quad b=q_{2} h_{01}^{2}, \quad c=q_{3} h_{10}^{2}, \quad d=q_{4} h_{11}^{2}
$$

Therefore

$$
\rho=\left(\begin{array}{cc}
\frac{\lambda-d}{c} \rho_{4} & 0 \\
0 & \rho_{4}
\end{array}\right)=\left(\begin{array}{cc}
\frac{b}{\lambda-a} \rho_{4} & 0 \\
0 & \rho_{4}
\end{array}\right)
$$

and

$$
\frac{\lambda-d}{c}=\frac{b}{\lambda-a}
$$

Solving for $\lambda$, we obtain the eigenvalues

$$
\lambda=\frac{a+d}{2} \pm \frac{\zeta}{2}=\frac{a+d}{2} \pm \frac{\sqrt{(d-a)^{2}+4 b c}}{2}
$$




$$
=\frac{1}{2}\left(q_{1} h_{00}^{2}+q_{4} h_{11}^{2} \pm \sqrt{\left(q_{4} h_{11}^{2}-q_{1} h_{00}^{2}\right)^{2}+4 q_{2} q_{3} h_{01}^{2} h_{10}^{2}}\right),
$$

where

$$
\zeta=\sqrt{(d-a)^{2}+4 b c}=\sqrt{\left(q_{4} h_{11}^{2}-q_{1} h_{00}^{2}\right)^{2}+4 q_{2} q_{3} h_{01}^{2} h_{10}^{2}}
$$

and the associated eigenfunctions

$$
\rho=\left(\begin{array}{cc}
\frac{a-d \pm \zeta}{2 c} \rho_{4} & 0 \\
0 & \rho_{4}
\end{array}\right)=\left(\begin{array}{cc}
\frac{2 b}{d-a \pm \zeta} \rho_{4} & 0 \\
0 & \rho_{4}
\end{array}\right)
$$

But $\rho_{1}+\rho_{4}=1$ so we obtain

$$
\begin{gathered}
\rho=\left(\begin{array}{cc}
\frac{a-d \pm \zeta}{a-d \pm \zeta+2 c} & 0 \\
0 & \frac{2 c}{a-d \pm \zeta+2 c}
\end{array}\right) \\
=\left(\begin{array}{cc}
\frac{q_{1} h_{00}^{2}-q_{4} h_{11}^{2} \pm \zeta}{q_{1} h_{00}^{2}-q_{4} h_{11}^{2} \pm \zeta+2 q_{3} h_{10}^{2}} & 0 \\
0 & \frac{2 q_{3} h_{10}^{2}}{q_{1} h_{00}^{2}-q_{4} h_{11}^{2} \pm \zeta+2 q_{3} h_{10}^{2}}
\end{array}\right)
\end{gathered}
$$

that is,

$$
\begin{gathered}
\rho=\left(\begin{array}{cc}
\frac{-2 b}{a-2 b-d \mp \zeta} & 0 \\
0 & \frac{a-d \mp \zeta}{a-2 b-d \mp \zeta}
\end{array}\right) \\
=\left(\begin{array}{cc}
\frac{-2 q_{2} h_{01}^{2}}{q_{1} h_{00}^{2}-2 q_{2} h_{01}^{2}-q_{4} h_{11}^{2} \mp \zeta} & 0 \\
0 & \frac{q_{1} h_{00}^{2}-q_{4} h_{11}^{2} \mp \zeta}{q_{1} h_{00}^{2}-2 q_{2} h_{01}^{2}-q_{4} h_{11}^{2} \mp \zeta}
\end{array}\right)
\end{gathered}
$$

Therefore we obtained that $\rho_{1}, \rho_{4}, q_{1}, \ldots, q_{4}, \lambda$ are implicit solutions for the set of equations (16)-(17). Recall that in this case we obtained $\rho_{2}=\rho_{3}=0$.

Now we consider the problem of finding the eigenvector associated to the dominant eigenvalue of $H$. The eigenvalues are

$$
\lambda=\frac{1}{2}\left(h_{00}+h_{11} \pm \sqrt{\left(h_{00}-h_{11}\right)^{2}+4 h_{01} h_{10}}\right)
$$

Then we can find $v$ such that $H v=\lambda v$ from the set of equations

$$
\begin{aligned}
& h_{00} v_{1}+h_{01} v_{2}=\lambda v_{1} \\
& h_{10} v_{1}+h_{11} v_{2}=\lambda v_{2}
\end{aligned}
$$

which determine $v_{1}, v_{2}, \lambda$ implicitly. Note that if we set

$$
q_{1}=\frac{1}{p_{00}}, \quad q_{2}=\frac{1}{p_{01}}, \quad q_{3}=\frac{1}{p_{10}}, \quad q_{4}=\frac{1}{p_{11}}
$$


we have that the set of equations (16)-(17) and (20)-(21) are the same. Hence we conclude that Perron's classic eigenvalue problem is a particular case of the problem for $\mathcal{L}$ acting on matrices.

\section{A theorem on eigenvalues for the Ruelle operator}

The following proposition is inspired in 25. We say that a hermitian operator $P: V \rightarrow V$ on a Hilbert space $(V,\langle\cdot\rangle)$ is positive if $\langle P v, v\rangle \geq 0$, for all $v \in V$, denoted $P \geq 0$. Consider the positive operator $\mathcal{L}_{W, V}: \mathcal{P} \mathcal{H}_{N} \rightarrow \mathcal{P} \mathcal{H}_{N}$,

$$
\mathcal{L}_{W, V}(\rho):=\sum_{i=1}^{k} \operatorname{tr}\left(W_{i} \rho W_{i}^{*}\right) V_{i} \rho V_{i}^{*}
$$

We have the following result:

Proposition 2 [1] There is $\rho \in \mathcal{M}_{N}$ and $\beta>0$ such that $\mathcal{L}_{W, V}(\rho)=\beta \rho$.

\section{Vector integrals and barycenters}

We recall here a few basic definitions. For more details, see [22] and 29]. Let $X$ be a metric space. Let $(V,+, \cdot)$ be a real vector space, and $\tau$ a topology on $V$. We say that $(V,+, \cdot ; \tau)$ is a topologic vector space if it is Hausdorff and if the operations + and $\cdot$ are continuous. For instance, in the context of density matrices, we will consider $V$ as the Hilbert space $\mathcal{H}_{N}$ and $X$ will be the space of density matrices $\mathcal{M}_{N}$.

Definition 3 Let $(X, \Sigma)$ be a measurable space, let $\mu \in M(X)$, let $(V,+, \cdot ; \tau)$ be a locally convex space and let $f: X \rightarrow V$. we say that $x \in V$ is the integral of $f$ in $X$, denoted by

$$
x:=\int_{X} f d \mu
$$

if

$$
\Psi(x)=\int_{X} \Psi \circ f d \mu
$$

for all $\Psi \in V^{*}$.

It is known that if we have a compact metric space $X, V$ is a locally convex space and $f: X \rightarrow V$ is a continuous function such that $\overline{c o} f(X)$ is compact 
then the integral of $f$ in $X$ exists and belongs to $\overline{c o} f(X)$. We will also use the following well-known result, the barycentric formula:

Proposition 3 [32] Let $V$ be a locally convex space, let $E \subset V$ be a complete, convex and bounded set, and $\mu \in M^{1}(E)$. Then there is a unique $x \in E$ such that

$$
l(x)=\int_{E} l d \mu,
$$

for all $l \in V^{*}$.

\section{Example: density matrices}

In this section we briefly review how the constructions of the previous section adjust to the case of density matrices.

Define $V:=\mathcal{H}_{N}, V^{+}:=\mathcal{P} \mathcal{H}_{N}$ (note that such space is a convex cone), and let the partial order $\leq$ on $\mathcal{P} \mathcal{H}_{N}$ be $\rho \leq \psi$ if and only if $\psi-\rho \geq 0$, i.e., if $\psi-\rho$ is positive. Then

$$
\left(V, V^{+}, e\right)=\left(\mathcal{H}_{N}, \mathcal{P} \mathcal{H}_{N}, t r\right),
$$

is a regular state space 29. Also, the set $B$ of unity trace in $V^{+}$is, of course, the space of density matrices. Hence, $B=\mathcal{M}_{N}$.

Let $Z \subset V^{*}$ be a nonempty vector subspace of $V^{*}$. The smallest topology in $V$ such that every functional defined in $Z$ is continuous on that topology, denoted by $\sigma(V, Z)$, turns $V$ into a locally convex space. In particular, $\sigma\left(V, V^{*}\right)$ is the weak topology in $V$. If $(V,\|\cdot\|)$ is a normed space, then $\sigma\left(V^{*}, V\right)$ is called a weak ${ }^{*}$ topology in $V^{*}$ (we identify $V$ with a subspace of $\left.V^{* *}\right)$. We also have that $(C, \tau)=\left(\mathcal{P} \mathcal{H}_{N}, \tau\right)$, where $\tau$ is the weak ${ }^{*}$ topology (and which is equal to the Euclidean, see [29]) is a metrizable compact structure. In this case we have that $B_{C}=B \cap C=\mathcal{M}_{N}$.

Definition $4 A$ Markov operator for probability measures is an operator $P: M^{1}(X) \rightarrow M^{1}(X)$ such that

$$
P\left(\lambda \mu_{1}+(1-\lambda) \mu_{2}\right)=\lambda P \mu_{1}+(1-\lambda) P \mu_{2},
$$

for $\mu_{1}, \mu_{2} \in M^{1}(X), \lambda \in(0,1)$.

An example of such an operator is one which we have defined before and we denote it $\mathcal{V}: M^{1}(X) \rightarrow M^{1}(X)$, 


$$
(\mathcal{V} \nu)(B)=\sum_{i=1}^{k} \int_{F_{i}^{-1}(B)} p_{i} d \nu,
$$

and we call it the Markov operator induced by the IFS $\mathcal{F}$. We will be interested in fixed points for $\mathcal{V}$.

Define

$$
m_{b}(X):=\{f: X \rightarrow \mathbb{R}: \mathrm{f} \text { is bounded, measurable }\}
$$

and also $\mathcal{U}: m_{b}(X) \rightarrow m_{b}(X)$,

$$
(\mathcal{U} f)(x):=\sum_{i=1}^{k} p_{i}(x) f\left(F_{i}(x)\right)
$$

Proposition 4 [29] Let $f \in m_{b}(X)$ and $\mu \in M^{1}(X)$, then

$$
\langle f, \mathcal{V} \mu\rangle=\langle\mathcal{U} f, \mu\rangle=\sum_{i=1}^{k} \int p_{i}\left(f \circ F_{i}\right) d \mu,
$$

where $\langle f, \mu\rangle$ denotes the integral of $f$ with respect to $\mu$.

Definition 5 An operator $Q: V^{+} \rightarrow V^{+}$is submarkovian if

1. $Q(x+y)=Q(x)+Q(y)$

2. $Q(\alpha x)=\alpha Q(x)$

3. $\|Q(x)\| \leq\|x\|$,

for all $x, y \in V^{+}, \alpha>0$.

Every submarkovian operator $Q: V^{+} \rightarrow V^{+}$can be extended in a unique way to a positive linear contraction on $V$.

Definition 6 Let $P: V^{+} \rightarrow V^{+}$a Markov operator and let $P_{i}: V^{+} \rightarrow V^{+}$, $i=1, \ldots, k$ be submarkovian operators such that $P=\sum_{i} P_{i}$. We say that $\left(P,\left\{P_{i}\right\}_{i=1}^{k}\right)$ is a Markov pair.

From [29], we know that there is a 1-1 correspondence between homogeneous IFS and Markov pairs.

Example 4 In this example we want to obtain a probability $\eta$ such that $\mathcal{V}(\eta)=\eta$.

Suppose a QIFS, such that

$$
p_{i}(\rho)=\operatorname{tr}\left(W_{i} \rho W_{i}^{*}\right), \quad \sum_{i} W_{i}^{*} W_{i}=I, \quad F_{i}(\rho)=\frac{V_{i} \rho V_{i}^{*}}{\operatorname{tr}\left(V_{i} \rho V_{i}^{*}\right)}
$$


for $i=1, \ldots, k$. Denote $m_{b}\left(\mathcal{M}_{N}\right)$ the space of bounded and measurable functions in $\mathcal{M}_{N}$. Consider $\Lambda: \mathcal{M}_{N} \rightarrow \mathcal{M}_{N}$,

$$
\Lambda(\rho)=\sum_{i} p_{i}(\rho) F_{i}(\rho)=\sum_{i} \operatorname{tr}\left(W_{i} \rho W_{i}^{*}\right) \frac{V_{i} \rho V_{i}^{*}}{\operatorname{tr}\left(V_{i} \rho V_{i}^{*}\right)}
$$

Suppose there exists a density matrix $\rho$ which $\Lambda$-invariant. As we know, such state is the barycenter of $\mu$ which is $\mathcal{V}$-invariant. Suppose $\mathcal{V} \mu=\mu$, then we can write

$$
\begin{aligned}
\int f d \mu=\int f d \mathcal{V} \mu & =\sum_{i=1}^{k} \int p_{i}(\rho) f\left(F_{i}(\rho)\right) d \mu(\rho)=\sum_{i} \int p_{i}(\rho) f\left(\frac{V_{i} \rho V_{i}^{*}}{\operatorname{tr}\left(V_{i} \rho V_{i}^{*}\right)}\right) d \mu \\
& =\sum_{i} \int \operatorname{tr}\left(W_{i} \rho W_{i}^{*}\right) f\left(\frac{V_{i} \rho V_{i}^{*}}{\operatorname{tr}\left(V_{i} \rho V_{i}^{*}\right)}\right) d \mu
\end{aligned}
$$

Therefore, for any $f \in m_{b}\left(\mathcal{M}_{N}\right)$, we got the condition

$$
\int f d \mu=\sum_{i} \int \operatorname{tr}\left(W_{i} \rho W_{i}^{*}\right) f\left(\frac{V_{i} \rho V_{i}^{*}}{\operatorname{tr}\left(V_{i} \rho V_{i}^{*}\right)}\right) d \mu
$$

Let us consider a particular example where $N=2, k=4$, and

$$
\begin{aligned}
& V_{1}=\left(\begin{array}{cc}
\sqrt{p_{11}} & 0 \\
0 & 0
\end{array}\right), \quad V_{2}=\left(\begin{array}{cc}
0 & \sqrt{p_{12}} \\
0 & 0
\end{array}\right), \\
& V_{3}=\left(\begin{array}{cc}
0 & 0 \\
\sqrt{p_{21}} & 0
\end{array}\right), \quad V_{4}=\left(\begin{array}{cc}
0 & 0 \\
0 & \sqrt{p_{22}}
\end{array}\right),
\end{aligned}
$$

in such way that the $p_{i j}$ are the entries of a column stochastic matrix P. Let $\pi=\left(\pi_{1}, \pi_{2}\right)$ be a vector such that $P \pi=\pi$. A simple calculation shows that for $\rho$, the density matrix such that has entries $\rho_{i j}$, we have

$$
\begin{aligned}
& V_{1} \rho V_{1}^{*}=\left(\begin{array}{cc}
p_{11} \rho_{11} & 0 \\
0 & 0
\end{array}\right), \quad V_{2} \rho V_{2}^{*}=\left(\begin{array}{cc}
p_{12} \rho_{22} & 0 \\
0 & 0
\end{array}\right) \\
& V_{3} \rho V_{3}^{*}=\left(\begin{array}{cc}
0 & 0 \\
0 & p_{21} \rho_{11}
\end{array}\right), \quad V_{4} \rho V_{4}^{*}=\left(\begin{array}{cc}
0 & 0 \\
0 & p_{22} \rho_{22}
\end{array}\right),
\end{aligned}
$$

and therefore

$$
\begin{gathered}
\frac{V_{1} \rho V_{1}^{*}}{\operatorname{tr}\left(V_{1} \rho V_{1}^{*}\right)}=\left(\begin{array}{ll}
1 & 0 \\
0 & 0
\end{array}\right), \frac{V_{2} \rho V_{2}^{*}}{\operatorname{tr}\left(V_{2} \rho V_{2}^{*}\right)}=\left(\begin{array}{ll}
1 & 0 \\
0 & 0
\end{array}\right) \\
\frac{V_{3} \rho V_{3}^{*}}{\operatorname{tr}\left(V_{3} \rho V_{3}^{*}\right)}=\left(\begin{array}{ll}
0 & 0 \\
0 & 1
\end{array}\right), \frac{V_{4} \rho V_{4}^{*}}{\operatorname{tr}\left(V_{4} \rho V_{4}^{*}\right)}=\left(\begin{array}{ll}
0 & 0 \\
0 & 1
\end{array}\right)
\end{gathered}
$$


that is, the above values do not depend on $\rho$.

Define

$$
\rho_{x}=\left(\begin{array}{ll}
1 & 0 \\
0 & 0
\end{array}\right), \quad \rho_{y}=\left(\begin{array}{ll}
0 & 0 \\
0 & 1
\end{array}\right)
$$

and

$$
\eta=\pi_{1} \delta_{\rho_{x}}+\pi_{2} \delta_{\rho_{y}}
$$

Note that the barycenter of $\eta$ is

$$
\rho_{\eta}=\pi_{1} \rho_{x}+\pi_{2} \rho_{y}=\pi_{1}\left(\begin{array}{ll}
1 & 0 \\
0 & 0
\end{array}\right)+\pi_{2}\left(\begin{array}{ll}
0 & 0 \\
0 & 1
\end{array}\right)=\left(\begin{array}{cc}
\pi_{1} & 0 \\
0 & \pi_{2}
\end{array}\right)
$$

For any mensurable set $B$ we have

$$
\mathcal{V} \eta(B)=\sum_{i=1}^{4} \int 1_{B}\left(F_{i}(\rho)\right) p_{i}(\rho) d \eta=\sum_{i=1}^{4} \int 1_{B}\left(\frac{V_{i} \rho V_{i}^{*}}{\operatorname{tr}\left(V_{i} \rho V_{i}^{*}\right)}\right) \operatorname{tr}\left(V_{i} \rho V_{i}^{*}\right) d \eta
$$

We can now consider the following cases:

1. Suppose first that $\rho_{x}, \rho_{y} \in B$. The using (25) and (26), one can show that

$$
\begin{gathered}
\mathcal{V} \eta(B)=\sum_{i=1}^{4} \rho_{11} \operatorname{tr}\left(V_{i} \rho_{x} V_{i}^{*}\right)+\rho_{22} \operatorname{tr}\left(V_{i} \rho_{y} V_{i}^{*}\right) \\
=\left(\pi_{1} p_{11}+0\right)+\left(0+\pi_{2} p_{12}\right)+\left(\pi_{1} p_{21}+0\right)+\left(0+\pi_{2} p_{22}\right)=\left(\pi_{1}+\pi_{2}\right)=1,
\end{gathered}
$$

because $P \pi=\pi$.

2. Suppose now that $\rho_{x} \in B, \rho_{y} \notin B$

$$
\mathcal{V} \eta(B)=\sum_{i=1}^{4} \pi_{1} \operatorname{tr}\left(V_{i} \rho_{x} V_{i}^{*}\right)=\pi_{1}\left(p_{11}+0+p_{21}+0\right)=\pi_{1}
$$

3. Finally, suppose that $\rho_{x} \notin B, \rho_{y} \in B$

$$
\mathcal{V} \eta(B)=\sum_{i=1}^{4} \pi_{2} \operatorname{tr}\left(V_{i} \rho_{y} V_{i}^{*}\right)=\pi_{2}\left(0+p_{12}+0+p_{22}\right)=\pi_{2}
$$

4. It is easy to see that if $\rho_{x}, \rho_{y} \notin B$ then $\mathcal{V} \eta(B)=0$.

The conclusion is that, $\mathcal{V} \eta(B)=\eta(B)$ for any measurable set $B$.

Therefore, $\mathcal{V}(\eta)=\eta$. 


\section{Some lemmas for IFS}

We want to understand the structure of $\Lambda: \mathcal{M}_{N} \rightarrow \mathcal{M}_{N}$,

$$
\Lambda(\rho):=\sum_{i=1}^{k} p_{i} F_{i}=\sum_{i=1}^{k} \operatorname{tr}\left(W_{i} \rho W_{i}^{*}\right) \frac{V_{i} \rho V_{i}^{*}}{\operatorname{tr}\left(V_{i} \rho V_{i}^{*}\right)}
$$

where $V_{i}, W_{i}$ are linear, $\sum_{i} W_{i}^{*} W_{i}=I$. Such operator is associated in a natural way to a IFS which is not homogeneous. In this section we state a few useful properties which are relevant for our study. The following lemmas hold for any IFS, except for lemma 3 for which a proof is known for homogeneous IFS only.

Lemma 1 Let $\left\{X, F_{i}, p_{i}\right\}_{i=1, \ldots, k}$ be a IFS, $\Psi$ a linear functional on $X$. Then $\mathcal{U} \circ \Psi=\Psi \circ \Lambda$.

Corollary 1 Let $\mathcal{F}=\left(X, F_{i}, p_{i}\right)_{i=1, \ldots, k}$ be a IFS and let $\rho_{0} \in X$. Then $\Lambda\left(\rho_{0}\right)=\rho_{0}$ if and only if $\mathcal{U}\left(\Psi\left(\rho_{0}\right)\right)=\Psi\left(\rho_{0}\right)$, for all $\Psi$ linear functional.

Lemma 2 Let $\mathcal{F}=\left\{X, F_{i}, p_{i}\right\}_{i=1, \ldots, k}$ be a IFS.

1. Let $\rho_{0} \in X$ such that $F_{i}\left(\rho_{0}\right)=\rho_{0}, i=1, \ldots, k$. Then $\mathcal{V} \delta_{\rho_{0}}=\delta_{\rho_{0}}$.

2. Let $\rho_{0} \in X$ such that $\mathcal{V} \delta_{\rho_{0}}=\delta_{\rho_{0}}$, then $\Lambda\left(\rho_{0}\right)=\rho_{0}$.

Lemma 3 Let $\left\{X, F_{i}, p_{i}\right\}_{i=1, \ldots, k}$ be a homogeneous IFS, $\Lambda=\sum_{i} p_{i} F_{i}$.

1. Let $\rho_{\nu}$ be the barycenter of a probability measure $\nu$. Then $\Lambda\left(\rho_{\nu}\right)$ is the barycenter of $\mathcal{V} \nu$, where $\mathcal{V}$ is the associated Markov operator.

2. Let $\mu$ be an invariant probability measure for $\mathcal{V}$. Then the barycenter of $\mu$, denoted by $\rho_{\mu}$, is a fixed point of $\Lambda$.

Example 5 Let $k=N=2$,

$$
V_{1}=\left(\begin{array}{cc}
-1 & 0 \\
0 & 1
\end{array}\right), \quad V_{2}=\left(\begin{array}{cc}
0 & -\frac{3 \sqrt{2}}{4} \\
-\frac{3 \sqrt{2}}{2} & 0
\end{array}\right),
$$

$W_{1}=(1 / 2) I, W_{2}=(\sqrt{3} / 2) I$. Then

$$
\begin{aligned}
& \Lambda(\rho)=\sum_{i} p_{i}(\rho) F_{i}(\rho)=\sum_{i} \operatorname{tr}\left(W_{i} \rho W_{i}^{*}\right) \frac{V_{i} \rho V_{i}^{*}}{\operatorname{tr}\left(V_{i} \rho V_{i}^{*}\right)} \\
= & \frac{1}{4} V_{1} \rho V_{1}^{*}+\frac{3}{4} \frac{V_{2} \rho V_{2}^{*}}{\operatorname{tr}\left(V_{2} \rho V_{2}^{*}\right)}=\frac{1}{4} V_{1} \rho V_{1}^{*}+\frac{3}{4} \frac{V_{2} \rho V_{2}^{*}}{\left(\frac{9}{8}+\frac{27}{8} \rho_{1}\right)}
\end{aligned}
$$

induces a IFS and it is such that $\rho_{0}=\frac{1}{3}|0\rangle\left\langle 0\left|+\frac{2}{3}\right| 1\right\rangle\langle 1|$ is a fixed point, with $F_{1}\left(\rho_{0}\right)=F_{2}\left(\rho_{0}\right)=\rho_{0}$. We can apply lemma 圆 and conclude that $\delta_{\rho_{0}}$ 
is an invariant measure for the Markov operator $\mathcal{V}$ associated to the IFS determined by $p_{i}$ and $F_{i}$.

The following lemma, a simple variation from results seen in [29], determines reasonable conditions that we will need in order to obtain a fixed point for $\mathcal{L}$ from a certain measure which is invariant for the Markov operator $\mathcal{V}$.

Lemma 4 Let $\left\{\mathcal{M}_{N}, F_{i}, p_{i}\right\}_{i=1, \ldots, k}$ be an IFS which admits an attractive invariant measure $\mu$ for $\mathcal{V}$. Then $\lim _{n \rightarrow \infty} \Lambda^{n}\left(\rho_{0}\right)=\rho_{\mu}$, for every $\rho_{0} \in \mathcal{M}_{N}$, where $\rho_{\mu}$ is the barycenter of $\mu$.

\section{Integral formulae for the entropy of IFS}

Part of the results we present here in this section are variations of the results presented in 29. Let $(X, d)$ be a complete separable metric space. Let $\left(V, V^{+}, e\right)$ be a complete state space, $B=\left\{x \in V^{+}: e(x)=1\right\}$ and $\mathcal{F}=\left(X, F_{i}, p_{i}\right)_{i=1, \ldots, k}$ the homogeneous IFS induced by the Markov pair $\left(\Lambda,\left\{\Lambda_{i}\right\}_{i=1}^{k}\right)$. Let $I_{k}=\{1, \ldots, k\}$ Let $n \in \mathbb{N}, \iota \in I_{k}^{n}, i \in I_{k}$. Define $F_{\iota i}:=F_{i} \circ F_{\iota}$ and

$$
p_{\iota i}(x)= \begin{cases}p_{i}\left(F_{\iota} x\right) p_{\iota}(x) & \text { if } p_{\iota}(x) \neq 0 \\ 0 & \text { otherwise }\end{cases}
$$

Proposition 5 Let $n \in \mathbb{N}, f \in m_{b}(X), x \in X$. Then

$$
\left(\mathcal{U}^{n} f\right)(x)=\sum_{\iota \in I_{k}^{n}} p_{\iota}(x) f\left(F_{\iota}(x)\right)
$$

Proposition 6 Let $x \in B, n \in \mathbb{N}$. Then

$$
\Lambda^{n}(x)=\sum_{\iota \in I_{k}^{n}} p_{\iota}(x) F_{\iota}(x) .
$$

Proposition 7 Let $\mathcal{F}$ be a IFS and let $g: B \rightarrow \mathbb{R}$. Then for $n \in \mathbb{N}$,

1. If $g$ is concave (resp. convex, affine) then $\mathcal{U}^{n} g \leq g \circ \Lambda^{n}$ (resp. $\mathcal{U}^{n} g \geq g \circ \Lambda^{n}$, $\left.\mathcal{U}^{n} g=g \circ \Lambda^{n}\right)$.

2. If $\bar{x}$ is a fixed point for $\Lambda$ then the sequence $\left.\left(\mathcal{U}^{n} g\right)(\bar{x})\right)_{n \in \mathbb{N}}$ is decreasing (resp. increasing, constant) if $g$ is concave (resp. convex, affine).

Also suppose that $\mathcal{F}$ is homogeneous. Then 
3. If $g$ is concave (resp. convex, affine), then $\mathcal{U} g$ is concave (resp. convex, affine).

Define $\eta: \mathbb{R}^{+} \rightarrow \mathbb{R}$ as

$$
\eta(x)= \begin{cases}-x \log x & \text { if } x \neq 0 \\ 0 & \text { if } x=0\end{cases}
$$

Define the Shannon-Boltzmann entropy function as $h: X \rightarrow \mathbb{R}^{+}$,

$$
h(x):=\sum_{i=1}^{k} \eta\left(p_{i}(x)\right)
$$

Let $n \in \mathbb{N}$. Define the partial entropy $H_{n}: X \rightarrow \mathbb{R}^{+}$as

$$
H_{n}(x):=\sum_{\iota \in I_{k}^{n}} \eta\left(p_{\iota}(x)\right),
$$

for $n \geq 1$ and $H_{0}(x):=0, x \in X$. Define, for $x \in X$,

$$
\overline{\mathcal{H}}(x):=\limsup _{n \rightarrow \infty} \frac{1}{n} H_{n}(x),
$$

the upper entropy on $\mathrm{x}$, and

$$
\underline{\mathcal{H}}(x):=\liminf _{n \rightarrow \infty} \frac{1}{n} H_{n}(x),
$$

the lower entropy on $\mathbf{x}$. If such limits are equal, we call its common value the entropy on $\mathbf{x}$, denoted by $\mathcal{H}(x)$.

Denote by $M^{\mathcal{V}}(X)$ the set of $\mathcal{V}$-invariant probability measures on $X$. Let $\mu \in M^{\mathcal{V}}(X)$. The partial entropy of the measure $\mu$ is defined by

$$
H_{n}(\mu):=\sum_{\iota \in I_{k}^{n}} \eta\left(\left\langle p_{\iota}, \mu\right\rangle\right)
$$

for $n \geq 1$ and $H_{0}(\mu):=0$.

Proposition 8 Let $\mu \in M^{\mathcal{V}}(X)$. Then the sequences $\left(\frac{1}{n} H_{n}(\mu)\right)_{n \in \mathbb{N}}$ and $\left(H_{n+1}(\mu)-H_{n}(\mu)\right)_{n \in \mathbb{N}}$ are nonnegative, decreasing, and have the same limit.

We denote the common limit of the sequences mentioned in the proposition above as $\mathcal{H}(\mu)$ and we call it the entropy of the measure $\mu$, i.e.,

$$
\mathcal{H}(\mu):=\lim _{n \rightarrow \infty} \frac{1}{n} H_{n}(\mu)=\lim _{n \rightarrow \infty}\left(H_{n+1}(\mu)-H_{n}(\mu)\right)
$$


The following result gives us an integral formula for entropy, and also a relation between the entropies defined before. We write $S(\mu):=M^{\mathcal{V}}(X) \cap$ $\operatorname{Lim}\left(\mathcal{V}^{n} \mu\right)_{n \in \mathbb{N}}$, where $\operatorname{Lim}\left(\mathcal{V}^{n} \mu\right)_{n \in \mathbb{N}}$ is the convex hull of the set of accumulation points of $\left(\mathcal{V}^{n} \mu\right)_{n \in \mathbb{N}}$, and $S_{\mathcal{F}}(\mu)$ is the set $S(\mu)$ associated to the Markov operator induced by the IFS $\mathcal{F}$. For the definition of compact structure and $(C, \tau)$-continuity, see [29].

Theorem 2 [29] (Integral formula for entropy of homogeneous IFS, compact case). Let $(C, \tau)$ be a metrizable compact structure $\left(V, V^{+}, e\right)$ such that $\left(\Lambda,\left\{\Lambda_{i}\right\}_{i=1}^{k}\right)$ is $(C, \tau)$-continuous. Assume that $\rho_{0} \in B_{C}:=B \cap C$ is such that $\Lambda\left(\rho_{0}\right)=\rho_{0}$. Then

$$
\mathcal{H}\left(\rho_{0}\right)=\mathcal{H}(\nu)=\int_{X} h d \nu
$$

for each $\nu \in S_{\mathcal{F}_{C}}\left(\delta_{\rho_{0}}\right)$, where $\mathcal{F}_{C}$ is the IFS $\mathcal{F}$ restricted to $\left(B_{C}, \tau\right)$.

The analogous result for hyperbolic IFS is the following.

Theorem 3 [29] Let $\mathcal{F}=\left(X, F_{i}, p_{i}\right)_{i=1, \ldots, k}$ be a hyperbolic IFS, $x \in X$, $\mu \in M^{1}(X)$ an invariant attractive measure for $\mathcal{F}$. Then

$$
\mathcal{H}(x)=\lim _{n \rightarrow \infty}\left(H_{n+1}(x)-H_{n}(x)\right)
$$

and

$$
\mathcal{H}(x)=\mathcal{H}(\mu)=\int_{X} h d \mu
$$

\section{Some calculations on entropy}

Let $U$ be a unitary matrix of order $m n$ acting on $\mathcal{H}_{m} \otimes \mathcal{H}_{n}$. Its Schmidt decomposition is

$$
U=\sum_{i=1}^{K} \sqrt{q_{i}} V_{i}^{A} \otimes V_{i}^{B}, \quad K=\min \left\{m^{2}, n^{2}\right\}
$$

The operators $V_{i}^{A}$ and $V_{i}^{B}$ act on certain Hilbert spaces $\mathcal{H}_{m}$ and $\mathcal{H}_{n}$, respectively. We also have that $\sum_{i=1}^{K} q_{i}=1$. Let $\sigma=\rho_{A} \otimes \rho_{*}^{B}=\rho_{A} \otimes I_{n} / n$ and define

$$
\Lambda\left(\rho_{A}\right):=\operatorname{tr}_{B}\left(U \sigma U^{*}\right)=\sum_{i=1}^{K} q_{i} V_{i}^{A} \rho_{A} V_{i}^{A *}
$$

Recall that 


$$
\operatorname{tr}_{B}\left(\left|a_{1}\right\rangle\left\langle a_{2}|\otimes| b_{1}\right\rangle\left\langle b_{2}\right|\right):=\left|a_{1}\right\rangle\left\langle a_{2}\right| \operatorname{tr}\left(\left|b_{1}\right\rangle\left\langle b_{2}\right|\right)
$$

where $\left|a_{1}\right\rangle$ and $\left|a_{2}\right\rangle$ are vectors on the state space of $A$ and $\left|b_{1}\right\rangle$ and $\left|b_{2}\right\rangle$ are vectors on the state space of $B$. The trace on the right side is the usual trace on $B$. A calculation shows that if $\rho_{*}^{A}=I_{m} / m$, then $\Lambda\left(\rho_{*}^{A}\right)=\rho_{*}^{A}$ and so $\Lambda$ is such that $\Lambda\left(I_{m} / m\right)=I_{m} / m$ and $\Lambda$ is trace preserving.

Let $\mathcal{F}$ be the homogeneous IFS associated to the $V_{i}^{A}$, that is, $p_{i}(\rho)=$ $\operatorname{tr}\left(q_{i} V_{i}^{A} \rho V_{i}^{A *}\right), F_{i}(\rho)=\left(q_{i} V_{i}^{A} \rho V_{i}^{A *}\right) / \operatorname{tr}\left(q_{i} V_{i}^{A} \rho V_{i}^{A *}\right)$ and let $\rho_{0}$ be a fixed point of $\Lambda=\sum_{i} p_{i} F_{i}$. Following [29, we have that $\rho_{0}$ is the barycenter of $\mathcal{V}^{n} \delta_{\rho_{0}}, n \in \mathbb{N}$. By theorem 2, we can calculate the entropy of such IFS. In this case we have

$$
\mathcal{H}\left(\rho_{0}\right)=\mathcal{H}(\nu)=\int_{\mathcal{M}_{N}} h d \nu
$$

where $\nu \in M^{\mathcal{V}}(X) \cap \operatorname{Lim}\left(\mathcal{V}^{n} \delta_{\rho_{0}}\right)_{n \in \mathbb{N}}$.

Let $\mathcal{F}=\left(\mathcal{M}_{N}, F_{i}, p_{i}\right)_{i=1, \ldots, k}$ be an IFS, $\Lambda(\rho)=\sum_{i} p_{i} F_{i}$. Let $\mathcal{U}$ be the conjugate of $\mathcal{V}$. By proposition 5

$$
\left(\mathcal{U}^{n} h\right)(\rho)=\sum_{\iota \in I_{k}^{n}(\rho)} p_{\iota}(\rho) h\left(F_{\iota}(\rho)\right)
$$

and since $h(\rho)=\sum_{j=1}^{k} \eta\left(p_{j}(\rho)\right)$, we have, for $\iota=\left(i_{1}, \ldots, i_{n}\right)$, and every $\rho_{0} \in \mathcal{M}_{N}$

$$
\begin{gathered}
\int_{\mathcal{M}_{N}} h d \mathcal{V}^{n} \delta_{\rho_{0}}=\int_{\mathcal{M}_{N}} \mathcal{U}^{n} h d \delta_{\rho_{0}} \\
=-\int_{\mathcal{M}_{N}} \sum_{\iota \in I_{k}^{n}(\rho)} p_{\iota}(\rho) \sum_{j=1}^{k} p_{j}\left(F_{\iota}(\rho)\right) \log p_{j}\left(F_{\iota}(\rho)\right) d \delta_{\rho_{0}} \\
=-\sum_{\iota \in I_{k}^{n}\left(\rho_{0}\right)} p_{\iota}\left(\rho_{0}\right) \sum_{j=1}^{k} p_{j}\left(F_{\iota}\left(\rho_{0}\right)\right) \log p_{j}\left(F_{\iota}\left(\rho_{0}\right)\right) \\
=-\sum_{\iota \in I_{k}^{n}\left(\rho_{0}\right)} p_{i_{1}}\left(\rho_{0}\right) p_{i_{2}}\left(F_{i_{1}} \rho_{0}\right) \cdots p_{i_{n}}\left(F_{i_{n-1}}\left(F_{i_{n-2}}\left(\cdots\left(F_{i_{1}} \rho_{0}\right)\right)\right)\right) \times \\
\times \sum_{j=1}^{k} p_{j}\left(F_{i_{n}}\left(F_{i_{n-1}}\left(\cdots\left(F_{i_{1}} \rho_{0}\right)\right)\right)\right) \log p_{j}\left(F_{i_{n}}\left(F_{i_{n-1}}\left(\cdots\left(F_{i_{1}} \rho_{0}\right)\right)\right)\right)=\left(\mathcal{U}^{n} h\right)\left(\rho_{0}\right)
\end{gathered}
$$

Suppose $\Lambda\left(\rho_{0}\right)=\rho_{0}$. We have by proposition 7 since $h$ is concave, that $\left(\mathcal{U}^{n} h\right)_{n \in \mathbb{N}}$ is decreasing, $\mathcal{U}^{n} h \leq h \circ \Lambda^{n}$ and so 


$$
\int_{\mathcal{M}_{N}} h d \mathcal{V}^{n} \delta_{\rho_{0}} \leq h\left(\Lambda^{n}\left(\rho_{0}\right)\right)=h\left(\rho_{0}\right)
$$

for every $n$.

\section{An expression for a stationary entropy}

In this section we present a definition of entropy which captures a stationary behavior.

Let $H$ be a hermitian operator and $V_{i}, i=1, \ldots, k$ linear operators. We can define the dynamics $F_{i}: \mathcal{M}_{N} \rightarrow \mathcal{M}_{N}$ :

$$
F_{i}(\rho):=\frac{V_{i} \rho V_{i}^{*}}{\operatorname{tr}\left(V_{i} \rho V_{i}^{*}\right)}
$$

Let $W_{i}, i=1, \ldots, k$ be linear and such that $\sum_{i=1}^{k} W_{i}^{*} W_{i}=I$. This determines functions $p_{i}: \mathcal{M}_{N} \rightarrow \mathbb{R}$,

$$
p_{i}(\rho):=\operatorname{tr}\left(W_{i} \rho W_{i}^{*}\right)
$$

Then we have $\sum_{i=1}^{k} p_{i}(\rho)=1$, for every $\rho$. Therefore a family $W:=$ $\left\{W_{i}\right\}_{i=1, \ldots, k}$ determines a QIFS $\mathcal{F}_{W}$,

$$
\mathcal{F}_{W}=\left\{\mathcal{M}_{N}, F_{i}, p_{i}\right\}_{i=1, \ldots, k}
$$

with $F_{i}, p_{i}$ given by (40) and (41).

Different choices of $W_{i}, i=1,2 \ldots, k$, as above, determine different invariant probabilities.

We introduce the following definition of entropy

Definition 7 Suppose that we have a QIFS such that there is a unique attractive invariant measure for the Markov operator $\mathcal{V}$ associated to $\mathcal{F}_{W}$. Let $\rho_{W}$ be the barycenter of such measure. Define

$h_{V}(W):=-\sum_{i=1}^{k} \operatorname{tr}\left(W_{i} \rho_{W} W_{i}^{*}\right) \sum_{j=1}^{k} \operatorname{tr}\left(\frac{W_{j} V_{i} \rho_{W} V_{i}^{*} W_{j}^{*}}{\operatorname{tr}\left(V_{i} \rho_{W} V_{i}^{*}\right)}\right) \log \operatorname{tr}\left(\frac{W_{j} V_{i} \rho_{W} V_{i}^{*} W_{j}^{*}}{\operatorname{tr}\left(V_{i} \rho_{W} V_{i}^{*}\right)}\right)$

Remember that by lemma 4 we have that $\rho_{W}$ is a fixed point for 


$$
\widehat{\mathcal{L}}_{\mathcal{F}_{W}}(\rho):=\sum_{i=1}^{k} p_{i}(\rho) F_{i}(\rho)=\sum_{i=1}^{k} \operatorname{tr}\left(W_{i} \rho W_{i}^{*}\right) \frac{V_{i} \rho V_{i}^{*}}{\operatorname{tr}\left(V_{i} \rho V_{i}^{*}\right)}
$$

Lemma 5 We have that $0 \leq h_{V}(W) \leq \log k$, for every family $W_{i}$ of linear operators satisfying $\sum_{i=1}^{k} W_{i}^{*} W_{i}=I$. Also, for any given dynamics $V$ the maximum can be reached.

We also define

$$
\mathcal{L}_{\mathcal{F}_{W}}(\rho):=\sum_{i=1}^{k} \operatorname{tr}\left(W_{i} \rho W_{i}^{*}\right) V_{i} \rho V_{i}^{*}
$$

Note that by the construction made on section 10, we have $h_{V}(W)=$ $\mathcal{U} h\left(\rho_{W}\right)$, where $\mathcal{U} h(\rho)=\sum_{i} p_{i}(\rho) h\left(F_{i}(\rho)\right)$.

Lemma 6 Let $\mathcal{F}=\left(\mathcal{M}_{N}, F_{i}, p_{i}\right)$ be a QIFS, with $F_{i}$, $p_{i}$ in the form 40) and [41). Suppose there is $\rho_{0} \in \mathcal{M}_{N}$ such that $\delta_{\rho_{0}}$ is the unique $\mathcal{V}$-invariant measure. Then $\widehat{\mathcal{L}}_{\mathcal{F}}\left(\rho_{0}\right)=\rho_{0}$ (eq. 43 ) $)$ and

$$
\int \mathcal{U}^{n} h d \delta_{\rho_{0}}=\mathcal{U}^{n} h\left(\rho_{0}\right)=h\left(\rho_{0}\right),
$$

for all $n \in \mathbb{N}$. Besides, $\mathcal{U}^{n} h\left(\rho_{0}\right)=\mathcal{U} h\left(\rho_{0}\right)$ and so

$$
h_{V}(W)=\mathcal{U}^{n} h\left(\rho_{0}\right),
$$

for all $n \in \mathbb{N}$.

Lemma 7 Let $\mu$ be a $\mathcal{V}$-invariant attractive measure. Then if $\rho_{\mu}$ is the barycenter of $\mu$ we have, for any $\rho$,

$$
\lim _{n \rightarrow \infty} \mathcal{U}^{n} h(\rho)=\int \mathcal{U} h d \mu=\int h d \mu \leq h\left(\rho_{\mu}\right)
$$

Lemma 8 Let $\mathcal{F}=\left(\mathcal{M}_{N}, F_{i}, p_{i}\right)$ be a QIFS, with $F_{i}, p_{i}$ in the form 40) and (41). Suppose that $\rho$ is the unique point such that $\widehat{\mathcal{L}}_{\mathcal{F}}(\rho)=\rho$. Suppose that $F_{i}(\rho)=\rho, i=1, \ldots, k$. Then

$$
\mathcal{U}^{n} h(\rho)=h(\rho),
$$

$n=1,2, \ldots$, and therefore $h_{V}(W)$ does not depend on $n$. 


\section{Entropy and Markov chains}

Let $V_{i}, W_{i}$ be linear operators, $i=1, \ldots, k, \sum_{i=1}^{k} W_{i}^{*} W_{i}=I$. Suppose the $V_{i}$ are fixed and determine a dynamics given by $F_{i}: \mathcal{M}_{N} \rightarrow \mathcal{M}_{N}, i=1, \ldots, k$. Define

$$
\begin{gathered}
P:=\left\{\left(p_{1}, \ldots, p_{k}\right): p_{i}: \mathcal{M}_{N} \rightarrow \mathbb{R}^{+}, i=1, \ldots, k, \sum_{i=1}^{k} p_{i}(\rho)=1, \forall \rho \in \mathcal{M}_{N}\right\} \\
P^{\prime}:=P \cap\left\{\left(p_{1}, \ldots, p_{k}\right): \exists W_{i}, i=1, \ldots, k: p_{i}(\rho)=\operatorname{tr}\left(W_{i} \rho W_{i}^{*}\right),\right. \\
\left.W_{i} \text { linear }, \sum_{i} W_{i}^{*} W_{i}=I\right\} \\
\mathcal{M}_{F}:=\left\{\mu \in M^{1}\left(\mathcal{M}_{N}\right): \exists p \in P^{\prime} \text { such that } \mathcal{V}_{p} \mu=\mu\right\},
\end{gathered}
$$

where $\mathcal{V}_{p}: M^{1}\left(\mathcal{M}_{N}\right) \rightarrow M^{1}\left(\mathcal{M}_{N}\right)$,

$$
\mathcal{V}_{p}(\mu)(B):=\sum_{i=1}^{k} \int_{F_{i}^{-1}(B)} p_{i} d \mu
$$

Note that a family $W:=\left\{W_{i}\right\}_{i=1, \ldots, k}$ determines a QIFS $\mathcal{F}_{W}$,

$$
\mathcal{F}_{W}=\left\{\mathcal{M}_{N}, F_{i}, p_{i}\right\}_{i=1, \ldots, k}
$$

As done in the previous section we introduce the following definition (which is in some sense stationary)

$$
h_{V}(W):=-\sum_{i=1}^{k} \frac{\operatorname{tr}\left(W_{i} \rho_{W} W_{i}^{*}\right)}{\operatorname{tr}\left(V_{i} \rho_{W} V_{i}^{*}\right)} \sum_{j=1}^{k} \operatorname{tr}\left(W_{j} V_{i} \rho_{W} V_{i}^{*} W_{j}^{*}\right) \log \left(\frac{\operatorname{tr}\left(W_{j} V_{i} \rho_{W} V_{i}^{*} W_{j}^{*}\right)}{\operatorname{tr}\left(V_{i} \rho_{W} V_{i}^{*}\right)}\right)
$$

where as before, $\rho_{W}$ denotes the barycenter of the unique attractive invariant measure for the Markov operator $\mathcal{V}$ associated to $\mathcal{F}_{W}$.

Let $P=\left(p_{i j}\right)_{i, j=1, \ldots, N}$ be a stochastic, irreducible matrix. Let $p$ be the stationary vector of $P$. The entropy of $P$ is defined as

$$
H(P):=-\sum_{i, j=1}^{N} p_{i} p_{i j} \log p_{i j}
$$

We consider an example which shows that the usual Markov chain entropy can be realized as the entropy associated to a certain QIFS.

Example 6 (Homogeneous case, 4 matrices). Let $N=2, k=4$ and 


$$
\begin{aligned}
& V_{1}=\left(\begin{array}{cc}
\sqrt{p_{00}} & 0 \\
0 & 0
\end{array}\right), \quad V_{2}=\left(\begin{array}{cc}
0 & \sqrt{p_{01}} \\
0 & 0
\end{array}\right), \\
& V_{3}=\left(\begin{array}{rr}
0 & 0 \\
\sqrt{p_{10}} & 0
\end{array}\right), \quad V_{4}=\left(\begin{array}{cc}
0 & 0 \\
0 & \sqrt{p_{11}}
\end{array}\right)
\end{aligned}
$$

Note that

$$
\sum_{i} V_{i}^{*} V_{i}=\left(\begin{array}{cc}
p_{00}+p_{10} & 0 \\
0 & p_{01}+p_{11}
\end{array}\right)
$$

and so $\sum_{i} V_{i}^{*} V_{i}=I$ if we suppose that

$$
P:=\left(\begin{array}{ll}
p_{00} & p_{01} \\
p_{10} & p_{11}
\end{array}\right)
$$

is column-stochastic. We have

$$
\begin{aligned}
& V_{1} \rho V_{1}^{*}=\left(\begin{array}{cc}
p_{00} \rho_{1} & 0 \\
0 & 0
\end{array}\right), \quad V_{2} \rho V_{2}^{*}=\left(\begin{array}{cc}
p_{01} \rho_{4} & 0 \\
0 & 0
\end{array}\right) \\
& V_{3} \rho V_{3}^{*}=\left(\begin{array}{cc}
0 & 0 \\
0 & p_{10} \rho_{1}
\end{array}\right), \quad V_{4} \rho V_{4}^{*}=\left(\begin{array}{cc}
0 & 0 \\
0 & p_{11} \rho_{4}
\end{array}\right)
\end{aligned}
$$

so

$$
\begin{gathered}
\operatorname{tr}\left(V_{1} \rho V_{1}^{*}\right)=p_{00} \rho_{1}, \quad \operatorname{tr}\left(V_{2} \rho V_{2}^{*}\right)=p_{01} \rho_{4} \\
\operatorname{tr}\left(V_{3} \rho V_{3}^{*}\right)=p_{10} \rho_{1}, \quad \operatorname{tr}\left(V_{4} \rho V_{4}^{*}\right)=p_{11} \rho_{4}
\end{gathered}
$$

The fixed point of $\Lambda(\rho)=\sum_{i} V_{i} \rho V_{i}^{*}$ is

$$
\rho_{V}=\left(\begin{array}{cc}
\frac{p_{01}}{1-p_{00}+p_{01}} & 0 \\
0 & \frac{1-p_{00}}{1-p_{00}+p_{01}}
\end{array}\right)
$$

Let $\pi=\left(\pi_{1}, \pi_{2}\right)$ such that $P \pi=\pi$. We know that

$$
\pi=\left(\frac{p_{01}}{1-p_{00}+p_{01}}, \frac{1-p_{00}}{1-p_{00}+p_{01}}\right)
$$

Then the nonzero entries of $\rho_{V}$ are the entries of $\pi$ and so we associate the fixed point of $P$ to the fixed point of a certain $\Lambda$ in a natural way. Let us calculate $h_{V}(W)$. Note that $\Lambda$ defined above is associated to a homogeneous IFS. Then $W_{i}=V_{i}, i=1, \ldots, k$ and

$$
h_{V}(W)=h_{V}(V)
$$




$$
\begin{gathered}
=-\sum_{i=1}^{k} \frac{\operatorname{tr}\left(W_{i} \rho_{V} W_{i}^{*}\right)}{\operatorname{tr}\left(V_{i} \rho_{V} V_{i}^{*}\right)} \sum_{j=1}^{k} \operatorname{tr}\left(W_{j} V_{i} \rho_{V} V_{i}^{*} W_{j}^{*}\right) \log \left(\frac{\operatorname{tr}\left(W_{j} V_{i} \rho_{V} V_{i}^{*} W_{j}^{*}\right)}{\operatorname{tr}\left(V_{i} \rho_{V} V_{i}^{*}\right)}\right) \\
=-\sum_{i, j} \operatorname{tr}\left(V_{j} V_{i} \rho_{V} V_{i}^{*} V_{j}^{*}\right) \log \left(\frac{\operatorname{tr}\left(V_{j} V_{i} \rho_{V} V_{i}^{*} V_{j}^{*}\right)}{\operatorname{tr}\left(V_{i} \rho_{V} V_{i}^{*}\right)}\right)
\end{gathered}
$$

A simple calculation yields $H(P)=h_{V}(V)$, where $H(P)$ is the entropy of $P$, given by (47). This shows that the entropy of Markov chains is a particular case of the entropy for QIFS defined before.

In a similar way, we can reach the same conclusion for the nonhomogeneous case, 4 matrices, and also for 2 matrices [1].

Lemma 9 Let $V_{i j}$ be matrices of order $n$,

$$
V_{i j}=\sqrt{p_{i j}}|i\rangle\langle j|
$$

for $i, j=1, \ldots, n$. Let

$$
\Lambda_{P}(\rho):=\sum_{i, j} V_{i j} \rho V_{i j}^{*}
$$

where $P=\left(p_{i j}\right)_{i, j=1, \ldots, n}$. Then for all $n, \Lambda_{P}^{n}(\rho)=\Lambda_{P^{n}}(\rho)$.

Corollary 2 Under the lemma hypothesis, we have $\lim _{n \rightarrow \infty} \Lambda_{P}^{n}(\rho)=\Lambda_{\pi}(\rho)$, where $\pi=\lim _{n \rightarrow \infty} P^{n}$ is the stochastic matrix which has all columns equal to the stationary vector for $P$.

\section{Capacity-cost function and pressure}

Recall that every trace preserving, completely positive (CP) mapping can be written in the Stinespring-Kraus form,

$$
\Lambda(\rho)=\sum_{i=1}^{k} V_{i} \rho V_{i}^{*}, \quad \sum_{i=1}^{k} V_{i}^{*} V_{i}=I,
$$

for $V_{i}$ linear operators. These mappings are also called quantum channels.

This is one of the main motivations for considering the class of operators (a generalization of the above ones) described in the present work. These are natural objets in the study of Quantum Computing. 
Definition 8 The Holevo capacity for sending classic information via a quantum channel $\Lambda$ is defined as

$$
C_{\Lambda}:=\max _{\substack{p_{i} \in[0,1] \\ \rho_{i} \in \mathcal{M}_{N}}} S\left(\sum_{i=1}^{n} p_{i} \Lambda\left(\rho_{i}\right)\right)-\sum_{i=1}^{n} p_{i} S\left(\Lambda\left(\rho_{i}\right)\right)
$$

where $S(\rho)=-\operatorname{tr}(\rho \log \rho)$ is the von Neumann entropy. The maximum is, therefore, over all choices of $p_{i}, i=1, \ldots, n$ and density operators $\rho_{i}$, for some $n \in \mathbb{N}$. The Holevo capacity establishes an upper bound on the amount of information that a quantum system contains [24].

Definition 9 Let $\Lambda$ be a quantum channel. Define the minimum output entropy as

$$
H^{\min }(\Lambda):=\min _{|\psi\rangle} S(\Lambda(|\psi\rangle\langle\psi|))
$$

Additivity conjecture We have that

$$
C_{\Lambda_{1} \otimes \Lambda_{2}}=C_{\Lambda_{1}}+C_{\Lambda_{2}}
$$

Minimum output entropy conjecture For any channels $\Lambda_{1}$ and $\Lambda_{2}$,

$$
H^{\min }\left(\Lambda_{1} \otimes \Lambda_{2}\right)=H^{\min }\left(\Lambda_{1}\right)+H^{\min }\left(\Lambda_{2}\right)
$$

In [27, is it shown that the additivity conjecture is equivalent to the minimum output entropy conjecture, and in [12] we obtain a counterexample for this last conjecture.

We will be interested here in a different class of problem which concern maximization (and not minimization) of entropy plus a given potential (a cost) [9], 13], [14.

Definition 10 Let $M_{F}$ be the set of invariant measures defined in the section 11 and let $H$ be a hermitian operator. For $\mu \in \mathcal{M}_{F}$ let $\rho_{\mu}$ be its barycenter. Define the capacity-cost function $C: \mathbb{R}^{+} \rightarrow \mathbb{R}^{+}$as

$$
C(a):=\max _{\mu \in \mathcal{M}_{F}}\left\{h_{W, V}\left(\rho_{\mu}\right): \operatorname{tr}\left(H \rho_{\mu}\right) \leq a\right\}
$$

The following analysis is inspired in [21]. There is a relation between the cost-capacity function and the variational problem for pressure. In fact, let $F: \mathbb{R}^{+} \rightarrow \mathbb{R}^{+}$be the function given by

$$
F(\lambda):=\sup _{\mu \in \mathcal{M}_{F}}\left\{h_{W, V}\left(\rho_{\mu}\right)-\lambda \operatorname{tr}\left(H \rho_{\mu}\right)\right\}
$$


We have the following fact. There is a unique probability measure $\nu_{0} \in \mathcal{M}_{F}$ such that

$$
F(\lambda)=h_{W, V}\left(\rho_{\nu_{0}}\right)-\lambda \operatorname{tr}\left(H \rho_{\nu_{0}}\right)
$$

Also, we have the following lemma:

Lemma 10 Let $\lambda \leq 0$, and $\hat{a}=\operatorname{tr}\left(H \rho_{\nu_{0}}\right)$. Then

$$
C(\hat{a})=h_{W, V}\left(\rho_{\nu_{0}}\right)
$$

\section{Analysis of the pressure problem}

Let $V_{i}, W_{i}$ be linear operators, $i=1, \ldots, k$, with $\sum_{i} W_{i}^{*} W_{i}=I$ and let

$$
H \rho:=\sum_{i=1}^{k} H_{i} \rho H_{i}^{*}
$$

a hermitian operator. We are interested in obtaining a version of the variational principle of pressure for our context. We will see that the pressure will be maximum whenever we have a certain relation between the potential $H$ and the probability distribution considered (and represented here by the $W_{i}$ ). Initially we consider that the $V_{i}$ are fixed. From the reasoning described below, it will be natural to consider as definition of pressure the maximization among the possible stationary $W_{i}$ of the expression

$$
h_{V}(W)+\sum_{j=1}^{k} \log \left(\operatorname{tr}\left(H_{j} \rho_{\beta} H_{j}^{*}\right) \operatorname{tr}\left(V_{j} \rho_{\beta} V_{j}^{*}\right)\right) \operatorname{tr}\left(W_{j} \rho_{W} W_{j}^{*}\right)
$$

Remember that different choices of $W_{i}, i=1,2, \ldots, k$, represent different choices of invariant probabilities.

Our analysis uses the following important lemma.

Lemma 11 If $r_{1}, \ldots, r_{k}$ and $q_{1}, \ldots, q_{k}$ are two probability distributions over $1, \ldots, k$, such that $r_{j}>0, j=1, \ldots, k$, then

$$
-\sum_{j=1}^{k} q_{j} \log q_{j}+\sum_{j=1}^{k} q_{j} \log r_{j} \leq 0
$$

and equality holds if and only if $r_{j}=q_{j}, j=1, \ldots, k$.

For the proof, see [25]. 
The potential given by (54) together with the $V_{i}$ induces an operator, given by

$$
\mathcal{L}_{H}(\rho):=\sum_{i=1}^{k} \operatorname{tr}\left(H_{i} \rho H_{i}^{*}\right) V_{i} \rho V_{i}^{*}
$$

We know that such operator admits an eigenvalue $\beta$ with its associate eigenstate $\rho_{\beta}$. Then $\mathcal{L}_{H}\left(\rho_{\beta}\right)=\beta \rho_{\beta}$ implies

$$
\sum_{i=1}^{k} \operatorname{tr}\left(H_{i} \rho_{\beta} H_{i}^{*}\right) V_{i} \rho_{\beta} V_{i}^{*}=\beta \rho_{\beta}
$$

In coordinates, (56) can be written as

$$
\sum_{i=1}^{k} \operatorname{tr}\left(H_{i} \rho_{\beta} H_{i}^{*}\right)\left(V_{i} \rho_{\beta} V_{i}^{*}\right)_{j l}=\beta\left(\rho_{\beta}\right)_{j l}
$$

Remark Comparing the above calculation with the problem of finding an eigenvalue $\lambda$ of a matrix $A=\left(a_{i j}\right)$, we have that equation (56) can be seen as the analogous of the expression

$$
l E^{A}=\lambda l
$$

Above, the matrix $A$ plays the role of a potential, $E^{A}$ denotes the matrix with entries $e^{a_{i j}}$ and $l_{j}$ denotes the $j$-th coordinate of the left eigenvector $l$ associated to the eigenvalue $\lambda$. In coordinates,

$$
\sum_{i} l_{i} e^{a_{i j}}=\lambda l_{j}, \quad i, j=1, \ldots, k
$$

From this point we can perform two calculations. First, considering (56) we will take the trace of such equation in order to obtain a scalar equation. In spite of the fact that taking the trace makes us lose part of the information given by the eigenvector equation, we are still able to obtain a version of what we will call a basic inequality, which can be seen as a quantum IFS version of the variational principle of pressure. However, there is an algebraic drawback to this approach, namely, that we will not be able to have the classic variational problem as a particular case of such inequality (such disadvantage is a consequence of taking the trace, clearly). The second calculation will consider (57), the coordinate equations associated to the matrix equation for the eigenvectors. In this case we also obtain a basic inequality, but now we will have the classic variational problem of pressure as a particular case. 
An important question which is of our interest, regarding both calculations mentioned above, is the question of whether it is possible for a given system to attain its maximum pressure. It is not clear that given any dynamics, we can obtain a measure reaching such a maximum. With respect to our context, we will state sufficient conditions on the dynamics which allows us to determine expressions for the measure which maximizes the pressure. We now perform the calculations mentioned above.

Based on (56), define

$$
r_{j}=\frac{1}{\beta} \operatorname{tr}\left(H_{j} \rho_{\beta} H_{j}^{*}\right) \operatorname{tr}\left(V_{j} \rho_{\beta} V_{j}^{*}\right)
$$

So we have $\sum_{j} r_{j}=1$. Let

$$
q_{j}^{i}:=\operatorname{tr}\left(\frac{W_{j} V_{i} \rho_{W} V_{i}^{*} W_{j}^{*}}{\operatorname{tr}\left(V_{i} \rho_{W} V_{i}^{*}\right)}\right)
$$

where, as before, $\rho_{W}$ is the fixed point associated to the renormalized operator $\Lambda_{\mathcal{F}_{W}}$,

$$
\Lambda_{\mathcal{F}_{W}}(\rho):=\sum_{i=1}^{k} p_{i}(\rho) F_{i}(\rho)
$$

induced by the QIFS $\left(\mathcal{M}_{N}, F_{i}, p_{i}\right)_{i=1, \ldots, k}$,

$$
F_{i}(\rho)=\frac{V_{i} \rho V_{i}^{*}}{\operatorname{tr}\left(V_{i} \rho V_{i}^{*}\right)}
$$

and

$$
p_{i}(\rho)=\operatorname{tr}\left(W_{i} \rho W_{i}^{*}\right)
$$

Note that we have

$$
\begin{gathered}
\sum_{j=1}^{k} q_{j}^{i}=\frac{1}{\operatorname{tr}\left(V_{i} \rho_{W} V_{i}^{*}\right)} \sum_{j=1}^{k} \operatorname{tr}\left(W_{j}^{*} W_{j} V_{i} \rho_{W} V_{i}^{*}\right) \\
=\frac{1}{\operatorname{tr}\left(V_{i} \rho_{W} V_{i}^{*}\right)} \operatorname{tr}\left(\sum_{j=1}^{k} W_{j}^{*} W_{j} V_{i} \rho_{W} V_{i}^{*}\right)=1
\end{gathered}
$$

Then we can apply lemma 11 for $r_{j}, q_{j}^{i}, j=1, \ldots k$, with $i$ fixed, to obtain

$$
-\sum_{j} \operatorname{tr}\left(\frac{W_{j} V_{i} \rho_{W} V_{i}^{*} W_{j}^{*}}{\operatorname{tr}\left(V_{i} \rho_{W} V_{i}^{*}\right)}\right) \log \operatorname{tr}\left(\frac{W_{j} V_{i} \rho_{W} V_{i}^{*} W_{j}^{*}}{\operatorname{tr}\left(V_{i} \rho_{W} V_{i}^{*}\right)}\right)
$$




$$
+\sum_{j} \operatorname{tr}\left(\frac{W_{j} V_{i} \rho_{W} V_{i}^{*} W_{j}^{*}}{\operatorname{tr}\left(V_{i} \rho_{W} V_{i}^{*}\right)}\right) \log \left(\frac{1}{\beta} \operatorname{tr}\left(H_{j} \rho_{\beta} H_{j}^{*}\right) \operatorname{tr}\left(V_{j} \rho_{\beta} V_{j}^{*}\right)\right) \leq 0
$$

and equality holds if and only if for all $i, j$,

$$
\frac{1}{\beta} \operatorname{tr}\left(H_{j} \rho_{\beta} H_{j}^{*}\right) \operatorname{tr}\left(V_{j} \rho_{\beta} V_{j}^{*}\right)=\frac{\operatorname{tr}\left(W_{j} V_{i} \rho_{W} V_{i}^{*} W_{j}^{*}\right)}{\operatorname{tr}\left(V_{i} \rho_{W} V_{i}^{*}\right)}
$$

Then

$$
\begin{gathered}
-\sum_{j} \operatorname{tr}\left(\frac{W_{j} V_{i} \rho_{W} V_{i}^{*} W_{j}^{*}}{\operatorname{tr}\left(V_{i} \rho_{W} V_{i}^{*}\right)}\right) \log \operatorname{tr}\left(\frac{W_{j} V_{i} \rho_{W} V_{i}^{*} W_{j}^{*}}{\operatorname{tr}\left(V_{i} \rho_{W} V_{i}^{*}\right)}\right) \\
+\sum_{j} \operatorname{tr}\left(\frac{W_{j} V_{i} \rho_{W} V_{i}^{*} W_{j}^{*}}{\operatorname{tr}\left(V_{i} \rho_{W} V_{i}^{*}\right)}\right) \log \left(\operatorname{tr}\left(H_{j} \rho_{\beta} H_{j}^{*}\right) \operatorname{tr}\left(V_{j} \rho_{\beta} V_{j}^{*}\right)\right) \\
\leq \sum_{j} \operatorname{tr}\left(\frac{W_{j} V_{i} \rho_{W} V_{i}^{*} W_{j}^{*}}{\operatorname{tr}\left(V_{i} \rho_{W} V_{i}^{*}\right)}\right) \log \beta
\end{gathered}
$$

which is equivalent to

$$
\begin{gathered}
-\sum_{j} \operatorname{tr}\left(\frac{W_{j} V_{i} \rho_{W} V_{i}^{*} W_{j}^{*}}{\operatorname{tr}\left(V_{i} \rho_{W} V_{i}^{*}\right)}\right) \log \operatorname{tr}\left(\frac{W_{j} V_{i} \rho_{W} V_{i}^{*} W_{j}^{*}}{\operatorname{tr}\left(V_{i} \rho_{W} V_{i}^{*}\right)}\right) \\
+\sum_{j} \frac{\operatorname{tr}\left(W_{j} V_{i} \rho_{W} V_{i}^{*} W_{j}^{*}\right)}{\operatorname{tr}\left(V_{i} \rho_{W} V_{i}^{*}\right)} \log \left(\operatorname{tr}\left(H_{j} \rho_{\beta} H_{j}^{*}\right) \operatorname{tr}\left(V_{j} \rho_{\beta} V_{j}^{*}\right)\right) \leq \log \beta
\end{gathered}
$$

Multiplying by $\operatorname{tr}\left(W_{i} \rho_{W} W_{i}^{*}\right)$ and summing over the $i$ index, we have

$$
\begin{gathered}
h_{V}(W)+\sum_{j} \log \left(\operatorname{tr}\left(H_{j} \rho_{\beta} H_{j}^{*}\right) \operatorname{tr}\left(V_{j} \rho_{\beta} V_{j}^{*}\right)\right) \sum_{i} \frac{\operatorname{tr}\left(W_{i} \rho_{W} W_{i}^{*}\right)}{\operatorname{tr}\left(V_{i} \rho_{W} V_{i}^{*}\right)} \operatorname{tr}\left(W_{j} V_{i} \rho_{W} V_{i}^{*} W_{j}^{*}\right) \\
\leq \sum_{i} \operatorname{tr}\left(W_{i} \rho_{W} W_{i}^{*}\right) \log \beta=\log \beta
\end{gathered}
$$

and equality holds if and only if for all $i, j$,

$$
\frac{1}{\beta} \operatorname{tr}\left(H_{j} \rho_{\beta} H_{j}^{*}\right) \operatorname{tr}\left(V_{j} \rho_{\beta} V_{j}^{*}\right)=\frac{\operatorname{tr}\left(W_{j} V_{i} \rho_{W} V_{i}^{*} W_{j}^{*}\right)}{\operatorname{tr}\left(V_{i} \rho_{W} V_{i}^{*}\right)}
$$

Let us rewrite inequality (66). First we use the fact that $\rho_{W}$ is a fixed point of $\Lambda_{\mathcal{F}_{W}}$,

$$
\sum_{i=1}^{k} \operatorname{tr}\left(W_{i} \rho_{W} W_{i}^{*}\right) \frac{V_{i} \rho_{W} V_{i}^{*}}{\operatorname{tr}\left(V_{i} \rho_{W} V_{i}^{*}\right)}=\rho_{W}
$$

Now we compose both sides of the equality above with the operator 


$$
\sum_{j=1}^{k} \log \left(\operatorname{tr}\left(H_{j} \rho_{\beta} H_{j}^{*}\right) \operatorname{tr}\left(V_{j} \rho_{\beta} V_{j}^{*}\right)\right) W_{j}^{*} W_{j}
$$

and then we obtain

$$
\begin{gathered}
\sum_{i=1}^{k} \operatorname{tr}\left(W_{i} \rho_{W} W_{i}^{*}\right) \frac{V_{i} \rho_{W} V_{i}^{*}}{\operatorname{tr}\left(V_{i} \rho_{W} V_{i}^{*}\right)} \sum_{j=1}^{k} \log \left(\operatorname{tr}\left(H_{j} \rho_{\beta} H_{j}^{*}\right) \operatorname{tr}\left(V_{j} \rho_{\beta} V_{j}^{*}\right)\right) W_{j}^{*} W_{j} \\
=\rho_{W} \sum_{j=1}^{k} \log \left(\operatorname{tr}\left(H_{j} \rho_{\beta} H_{j}^{*}\right) \operatorname{tr}\left(V_{j} \rho_{\beta} V_{j}^{*}\right)\right) W_{j}^{*} W_{j}
\end{gathered}
$$

Reordering terms we get

$$
\begin{gathered}
\sum_{j=1}^{k} \log \left(\operatorname{tr}\left(H_{j} \rho_{\beta} H_{j}^{*}\right) \operatorname{tr}\left(V_{j} \rho_{\beta} V_{j}^{*}\right)\right) \sum_{i=1}^{k} \frac{\operatorname{tr}\left(W_{i} \rho_{W} W_{i}^{*}\right)}{\operatorname{tr}\left(V_{i} \rho_{W} V_{i}^{*}\right)} V_{i} \rho_{W} V_{i}^{*} W_{j}^{*} W_{j} \\
=\rho_{W} \sum_{j=1}^{k} \log \left(\operatorname{tr}\left(H_{j} \rho_{\beta} H_{j}^{*}\right) \operatorname{tr}\left(V_{j} \rho_{\beta} V_{j}^{*}\right)\right) W_{j}^{*} W_{j}
\end{gathered}
$$

Taking the trace on both sides we get

$$
\begin{gathered}
\sum_{j=1}^{k} \log \left(\operatorname{tr}\left(H_{j} \rho_{\beta} H_{j}^{*}\right) \operatorname{tr}\left(V_{j} \rho_{\beta} V_{j}^{*}\right)\right) \sum_{i=1}^{k} \frac{\operatorname{tr}\left(W_{i} \rho_{W} W_{i}^{*}\right)}{\operatorname{tr}\left(V_{i} \rho_{W} V_{i}^{*}\right)} \operatorname{tr}\left(W_{j} V_{i} \rho_{W} V_{i}^{*} W_{j}^{*}\right) \\
=\sum_{j=1}^{k} \log \left(\operatorname{tr}\left(H_{j} \rho_{\beta} H_{j}^{*}\right) \operatorname{tr}\left(V_{j} \rho_{\beta} V_{j}^{*}\right)\right) \operatorname{tr}\left(\rho_{W} W_{j}^{*} W_{j}\right)
\end{gathered}
$$

Note that the left hand side of (72) is one of the sums appearing in (66). Therefore replacing (72) into (66) gives us the following inequality:

$$
h_{V}(W)+\sum_{j=1}^{k} \log \left(\operatorname{tr}\left(H_{j} \rho_{\beta} H_{j}^{*}\right) \operatorname{tr}\left(V_{j} \rho_{\beta} V_{j}^{*}\right)\right) \operatorname{tr}\left(W_{j} \rho_{W} W_{j}^{*}\right) \leq \log \beta
$$

and equality holds if and only if for all $i, j$,

$$
\frac{1}{\beta} \operatorname{tr}\left(H_{j} \rho_{\beta} H_{j}^{*}\right) \operatorname{tr}\left(V_{j} \rho_{\beta} V_{j}^{*}\right)=\frac{\operatorname{tr}\left(W_{j} V_{i} \rho_{W} V_{i}^{*} W_{j}^{*}\right)}{\operatorname{tr}\left(V_{i} \rho_{W} V_{i}^{*}\right)}
$$

So we have the following result.

Theorem 4 Let $\mathcal{F}_{W}$ be a QIFS such that there is a unique attractive invariant measure for the associated Markov operator $\mathcal{V}$. Let $\rho_{W}$ be the barycenter of such measure and let $\rho_{\beta}$ be an eigenstate of $\mathcal{L}_{H}(\rho)$ with eigenvalue $\beta$. Then 


$$
h_{V}(W)+\sum_{j=1}^{k} \log \left(\operatorname{tr}\left(H_{j} \rho_{\beta} H_{j}^{*}\right) \operatorname{tr}\left(V_{j} \rho_{\beta} V_{j}^{*}\right)\right) \operatorname{tr}\left(W_{j} \rho_{W} W_{j}^{*}\right) \leq \log \beta
$$

and equality holds if and only if for all $i, j$,

$$
\frac{1}{\beta} \operatorname{tr}\left(H_{j} \rho_{\beta} H_{j}^{*}\right) \operatorname{tr}\left(V_{j} \rho_{\beta} V_{j}^{*}\right)=\frac{\operatorname{tr}\left(W_{j} V_{i} \rho_{W} V_{i}^{*} W_{j}^{*}\right)}{\operatorname{tr}\left(V_{i} \rho_{W} V_{i}^{*}\right)}
$$

In section [15] we make some considerations about certain cases in which we can reach an equality in (75).

For the calculations regarding expression (57), define

$$
r_{j l m}=\frac{1}{\beta} \operatorname{tr}\left(H_{j} \rho_{\beta} H_{j}^{*}\right) \frac{\left(V_{j} \rho_{\beta} V_{j}^{*}\right)_{l m}}{\left(\rho_{\beta}\right)_{l m}}
$$

Then we have $\sum_{j} r_{j l m}=1$. Let

$$
q_{i j}:=\operatorname{tr}\left(\frac{W_{j} V_{i} \rho_{W} V_{i}^{*} W_{j}^{*}}{\operatorname{tr}\left(V_{i} \rho_{W} V_{i}^{*}\right)}\right)
$$

A calculation similar to the one we have made for (75) gives us

$$
\begin{gathered}
h_{V}(W)+\sum_{j=1}^{k} \operatorname{tr}\left(W_{j} \rho_{W} W_{j}^{*}\right) \log \operatorname{tr}\left(H_{j} \rho_{\beta} H_{j}^{*}\right) \\
+\sum_{j=1}^{k} \operatorname{tr}\left(W_{j} \rho_{W} W_{j}^{*}\right) \log \left(\frac{\left(V_{j} \rho_{\beta} V_{j}^{*}\right)_{l m}}{\left(\rho_{\beta}\right)_{l m}}\right) \leq \log \beta
\end{gathered}
$$

and equality holds if and only if for all $i, j, l, m$,

$$
\frac{1}{\beta} \operatorname{tr}\left(H_{j} \rho_{\beta} H_{j}^{*}\right) \frac{\left(V_{j} \rho_{\beta} V_{j}^{*}\right)_{l m}}{\left(\rho_{\beta}\right)_{l m}}=\frac{\operatorname{tr}\left(W_{j} V_{i} \rho_{W} V_{i}^{*} W_{j}^{*}\right)}{\operatorname{tr}\left(V_{i} \rho_{W} V_{i}^{*}\right)}
$$

\section{Some classic inequality calculations}

A natural question is to ask whether the maximum among normalized $W_{i}$, $i=1, \ldots, k$, for the pressure problem associated to a given potential is realized as the logarithm of the main eigenvalue of a certain Ruelle operator associated 
to the potential $H_{i}, i=1, \ldots, k$. This problem will be considered in this section and also in the next one.

We begin by recalling a classic inequality. Consider

$$
-\sum_{j=1}^{k} q_{j} \log q_{j}+\sum_{j=1}^{k} q_{j} \log r_{j} \leq 0
$$

given by lemma 11. Let $A$ be a matrix. If $v$ denotes the left eigenvector of matrix $E^{A}$ (such that each entry is $e^{a_{i j}}$ ), then $v E^{A}=\beta v$ can be written as

$$
\sum_{i} v_{i} e^{a_{i j}}=\beta v_{j}, \quad \forall j
$$

Define

$$
r_{i j}:=\frac{e^{a_{i j}} v_{i}}{\beta v_{j}}
$$

So $\sum_{i} r_{i j}=1$. Let $q_{i j}>0$ such that $\sum_{i} q_{i j}=1$. By (81), we have

$$
-\sum_{i=1}^{k} q_{i j} \log q_{i j}+\sum_{i=1}^{k} q_{i j} \log \frac{e^{a_{i j}} v_{i}}{\beta v_{j}} \leq 0
$$

That is,

$$
-\sum_{i=1}^{k} q_{i j} \log q_{i j}+\sum_{i=1}^{k} q_{i j} a_{i j}+\sum_{i=1}^{k} q_{i j}\left(\log v_{i}-\log v_{j}\right) \leq \log \beta
$$

Let $Q$ be a matrix with entries $q_{i j}$, let $\pi=\left(\pi_{1}, \ldots, \pi_{k}\right)$ be the stationary vector associated to $Q$. Since $\sum_{i} q_{i j}=1, Q$ is column-stochastic so we write $Q \pi=\pi$. Multiplying the above inequality by $\pi_{j}$ and summing the $j$ index, we get

$$
-\sum_{j} \pi_{j} \sum_{i} q_{i j} \log q_{i j}+\sum_{j} \pi_{j} \sum_{i} q_{i j} a_{i j}+\sum_{j} \pi_{j} \sum_{i} q_{i j}\left(\log v_{i}-\log v_{j}\right) \leq \log \beta
$$

In coordinates, $Q \pi=\pi$ is $\sum_{j} q_{i j} \pi_{j}=\pi_{i}$, for all $i$. Then

$$
\begin{gathered}
-\sum_{j} \pi_{j} \sum_{i} q_{i j} \log q_{i j}+\sum_{j} \pi_{j} \sum_{i} q_{i j} a_{i j} \\
+\sum_{j} \pi_{j} \sum_{i} q_{i j} \log v_{i}-\sum_{j} \pi_{j} \sum_{i} q_{i j} \log v_{j} \leq \log \beta
\end{gathered}
$$

These calculations are well-known and give the following inequality: 


$$
-\sum_{j} \pi_{j} \sum_{i} q_{i j} \log q_{i j}+\sum_{j} \pi_{j} \sum_{i} q_{i j} a_{i j} \leq \log \beta
$$

Definition 11 We call inequality (88) the classic inequality associated to the matrix $A$ with positive entries, and stochastic matrix $Q$.

Definition 12 For fixed $k$, and $l, m=1, \ldots, k$ we call the inequality

$$
\begin{gathered}
h_{V}(W)+\sum_{j=1}^{k} \operatorname{tr}\left(W_{j} \rho_{W} W_{j}^{*}\right) \log \operatorname{tr}\left(H_{j} \rho_{\beta} H_{j}^{*}\right) \\
+\sum_{j=1}^{k} \operatorname{tr}\left(W_{j} \rho_{W} W_{j}^{*}\right) \log \left(\frac{\left(V_{j} \rho_{\beta} V_{j}^{*}\right)_{l m}}{\left(\rho_{\beta}\right)_{l m}}\right) \leq \log \beta,
\end{gathered}
$$

the basic inequality associated to the potential $H \rho=\sum_{i} H_{i} \rho H_{i}^{*}$ and to the QIFS determined by $V_{i}, W_{i}, i=1, \ldots, k$. Equality holds if for all $i, j, l, m$,

$$
\frac{1}{\beta} \operatorname{tr}\left(H_{j} \rho_{\beta} H_{j}^{*}\right) \frac{\left(V_{j} \rho_{\beta} V_{j}^{*}\right)_{l m}}{\left(\rho_{\beta}\right)_{l m}}=\frac{\operatorname{tr}\left(W_{j} V_{i} \rho_{W} V_{i}^{*} W_{j}^{*}\right)}{\operatorname{tr}\left(V_{i} \rho_{W} V_{i}^{*}\right)}
$$

As before $\rho_{\beta}$ is an eigenstate of $\mathcal{L}_{H}(\rho)$ and $\rho_{W}$ is the barycenter of the unique attractive, invariant measure for the Markov operator $\mathcal{V}$ associated to the QIFS $\mathcal{F}_{W}$. Given the classic inequality (88) we want to compare it to the basic inequality (89). More precisely, we would like to obtain operators $V_{i}$ that satisfy the following: given a matrix $A$ with positive entries and a stochastic matrix $Q$, there are $H_{i}$ and $W_{i}$ such that inequality (89) becomes inequality (88). We have the following proposition.

Proposition 9 [1] Define

$$
\begin{aligned}
& V_{1}=\left(\begin{array}{ll}
1 & 0 \\
0 & 0
\end{array}\right), \quad V_{2}=\left(\begin{array}{ll}
0 & 1 \\
0 & 0
\end{array}\right) \\
& V_{3}=\left(\begin{array}{ll}
0 & 0 \\
1 & 0
\end{array}\right), \quad V_{4}=\left(\begin{array}{ll}
0 & 0 \\
0 & 1
\end{array}\right)
\end{aligned}
$$

Let $A=\left(a_{i j}\right)$ be a matrix with positive entries and $Q=\left(q_{i j}\right)$ a twodimensional column-stochastic matrix. Define

$$
\begin{aligned}
& H_{11}=\left(\begin{array}{cc}
\sqrt{e^{a_{11}}} & \sqrt{e^{a_{11}}} \\
0 & 0
\end{array}\right), H_{12}=\left(\begin{array}{cc}
\sqrt{e^{a_{12}}} & \sqrt{e^{a_{12}}} \\
0 & 0
\end{array}\right) \\
& H_{21}=\left(\begin{array}{cc}
0 & 0 \\
\sqrt{e^{a_{21}}} & \sqrt{e^{a_{21}}}
\end{array}\right), H_{22}=\left(\begin{array}{cc}
0 & 0 \\
\sqrt{e^{a_{22}}} & \sqrt{e^{a_{22}}}
\end{array}\right)
\end{aligned}
$$


and also

$$
\begin{aligned}
& W_{1}=\left(\begin{array}{cc}
\sqrt{q_{11}} & 0 \\
0 & 0
\end{array}\right), W_{2}=\left(\begin{array}{cc}
0 & \sqrt{q_{12}} \\
0 & 0
\end{array}\right) \\
& W_{3}=\left(\begin{array}{cc}
0 & 0 \\
\sqrt{q_{21}} & 0
\end{array}\right), W_{4}=\left(\begin{array}{cc}
0 & 0 \\
0 & \sqrt{q_{22}}
\end{array}\right)
\end{aligned}
$$

Then the basic inequality associated to $W_{i}, V_{i}, H_{i}, i=1, \ldots, 4, l=m=1$ or $l=m=2$, is equivalent to the classic inequality associated to $A$ and $Q$.

\section{Example 7 Let}

$$
H_{1}=\left(\begin{array}{cc}
2 i & 2 i \\
0 & 0
\end{array}\right), \quad H_{2}=I, \quad H_{3}=\left(\begin{array}{cc}
i \sqrt{2} & i \sqrt{2} \\
0 & 0
\end{array}\right), \quad H_{4}=I
$$

Then

$$
H_{1}^{*}=\left(\begin{array}{ll}
-2 i & 0 \\
-2 i & 0
\end{array}\right), \quad H_{2}^{*}=I, \quad H_{3}^{*}=\left(\begin{array}{ll}
-i \sqrt{2} & 0 \\
-i \sqrt{2} & 0
\end{array}\right), \quad H_{4}^{*}=I
$$

If we suppose the $V_{i}$ are the same as from proposition 9, we have that $\rho_{\beta}$ is diagonal, so

$$
\operatorname{tr}\left(H_{1} \rho_{\beta} H_{1}^{*}\right)=4, \operatorname{tr}\left(H_{2} \rho_{\beta} H_{2}^{*}\right)=1, \operatorname{tr}\left(H_{3} \rho_{\beta} H_{3}^{*}\right)=2, \operatorname{tr}\left(H_{4} \rho_{\beta} H_{4}^{*}\right)=1
$$

Then $\mathcal{L}_{H}(\rho)=\beta \rho$ leads us to

$$
\begin{aligned}
& 4 \rho_{11}+\rho_{22}=\beta \rho_{11} \\
& 2 \rho_{11}+\rho_{22}=\beta \rho_{22}
\end{aligned}
$$

A simples calculation gives

$$
\beta=\frac{5+\sqrt{17}}{2}
$$

with eigenstate

$$
\rho_{\beta}=\frac{4}{7+\sqrt{17}}\left(\begin{array}{cc}
\frac{3+\sqrt{17}}{4} & 0 \\
0 & 1
\end{array}\right)
$$

We want to calculate the $W_{i}$ which maximize the basic inequality (89). Recall that from proposition 9 , the choice of $V_{i}$ we made is such that

$$
\frac{\left(V_{j} \rho_{\beta} V_{j}^{*}\right)_{l m}}{\left(\rho_{\beta}\right)_{l m}}=1,
$$

So

$$
h_{V}(W)+\sum_{j=1}^{k} \operatorname{tr}\left(W_{j} \rho_{W} W_{j}^{*}\right) \log \operatorname{tr}\left(H_{j} \rho_{\beta} H_{j}^{*}\right) \leq \log \beta
$$


and equality holds if and only if, for all $i, j, l, m$,

$$
\frac{1}{\beta} \operatorname{tr}\left(H_{j} \rho_{\beta} H_{j}^{*}\right) \frac{\left(V_{j} \rho_{\beta} V_{j}^{*}\right)_{l m}}{\left(\rho_{\beta}\right)_{l m}}=\frac{\operatorname{tr}\left(W_{j} V_{i} \rho_{W} V_{i}^{*} W_{j}^{*}\right)}{\operatorname{tr}\left(V_{i} \rho_{W} V_{i}^{*}\right)}
$$

Choose, for instance, $l=m=1$. Then condition (98) becomes

$$
\frac{1}{\beta} \operatorname{tr}\left(H_{j} \rho_{\beta} H_{j}^{*}\right)=\frac{\operatorname{tr}\left(W_{j} V_{i} \rho_{W} V_{i}^{*} W_{j}^{*}\right)}{\operatorname{tr}\left(V_{i} \rho_{W} V_{i}^{*}\right)}
$$

To simplify calculations, write $\widehat{W}_{i}=W_{i}^{*} W_{i}$ and $\widehat{W}_{i}=\left(w_{i j}^{i}\right)$. Then we get

$$
\frac{\operatorname{tr}\left(H_{i} \rho_{\beta} H_{i}^{*}\right)}{\beta}=w_{11}^{i}=w_{22}^{i}, \quad i=1, \ldots, 4
$$

So we conclude

$$
W_{i}=\frac{1}{\sqrt{\beta}}\left(\begin{array}{cc}
\sqrt{\operatorname{tr}\left(H_{i} \rho_{\beta} H_{i}^{*}\right)} & 0 \\
0 & \sqrt{\operatorname{tr}\left(H_{i} \rho_{\beta} H_{i}^{*}\right)}
\end{array}\right), \quad i=1, \ldots, 4
$$

That is,

$$
W_{1}=\frac{2}{\sqrt{\beta}} I, \quad W_{2}=\frac{1}{\sqrt{\beta}} I, \quad W_{3}=\frac{\sqrt{2}}{\sqrt{\beta}} I, \quad W_{4}=\frac{1}{\sqrt{\beta}} I
$$

Note that

$$
\sum_{i} W_{i}^{*} W_{i}=\frac{4+\sqrt{2}}{\sqrt{\beta}} I \neq I
$$

To solve that, we renormalize the potential. Define

$$
\tilde{H}_{i}:=\sqrt{\alpha} H_{i}
$$

where

$$
\alpha:=\frac{\sqrt{\beta}}{4+\sqrt{2}}
$$

Then a calculation shows that $\mathcal{L}_{\tilde{H}}(\rho)=\tilde{\beta} \rho$ gives us the same eigenstate as before, that is $\rho_{\tilde{\beta}}=\rho_{\beta}$. But note that the associated eigenvalue becomes $\tilde{\beta}=\alpha \beta$. Now, note that it is possible to renormalize the $W_{i}$ in such a way that we obtain $\tilde{W}_{i}$ with $\sum_{i} \tilde{W}_{i}^{*} \tilde{W}_{i}=I$, and that these maximize the basic inequality for the $H_{i}$ initially fixed. In fact, given the renormalized $\tilde{H}_{i}$, define

$$
\tilde{W}_{i}=\sqrt{\alpha} W_{i}, \quad i=1, \ldots, 4
$$

Note that $\sum_{i} \tilde{W}_{i}^{*} \tilde{W}_{i}=I$. Also we obtain 


$$
h_{V}(\tilde{W})+\sum_{j=1}^{k} \operatorname{tr}\left(\tilde{W}_{j} \rho_{\tilde{W}} \tilde{W}_{j}^{*}\right) \log \operatorname{tr}\left(\sqrt{\alpha} H_{j} \rho_{\beta} \sqrt{\alpha} H_{j}^{*}\right) \leq \log \alpha \beta
$$

which is equivalent to

$$
h_{V}(\tilde{W})+\sum_{j=1}^{k} \operatorname{tr}\left(\tilde{W}_{j} \rho_{\tilde{W}} \tilde{W}_{j}^{*}\right) \log \left(\alpha \operatorname{tr}\left(H_{j} \rho_{\beta} H_{j}^{*}\right)\right) \leq \log \alpha+\log \beta
$$

That is

$$
\begin{gathered}
h_{V}(\tilde{W})+\sum_{j=1}^{k} \operatorname{tr}\left(\tilde{W}_{j} \rho_{\tilde{W}} \tilde{W}_{j}^{*}\right) \log \alpha \\
+\sum_{j=1}^{k} \operatorname{tr}\left(\tilde{W}_{j} \rho_{\tilde{W}} \tilde{W}_{j}^{*}\right) \log \operatorname{tr}\left(H_{j} \rho_{\beta} H_{j}^{*}\right) \leq \log \alpha+\log \beta,
\end{gathered}
$$

and cancelling $\log \alpha$, we get the same inequality as for the nonrenormalized $H_{i}$. As we have seen before, such $\tilde{W}_{i}$ gives us equality. Hence

$$
h_{V}(\tilde{W})+\sum_{j=1}^{k} \operatorname{tr}\left(\tilde{W}_{j} \rho_{\tilde{W}} \tilde{W}_{j}^{*}\right) \log \operatorname{tr}\left(H_{j} \rho_{\beta} H_{j}^{*}\right)=\log \beta
$$

\section{Remarks on the problem of pressure and quantum mechanics}

One of the questions we are interested in is to understand how to formulate a variational principle for pressure in the context of quantum information theory. An appropriate combination of such theories could have as a starting point a relation between the inequality for positive numbers

$$
-\sum_{i} q_{i} \log q_{i}+\sum_{i} q_{i} \log p_{i} \leq 0,
$$

(seen in certain proofs of the variational principle of pressure), and the entropy for QIFS we defined before. We have carried out such a plan and then we have obtained the basic inequality, which can be written as

$$
h_{V}(W)+\sum_{j=1}^{k} \log \left(\operatorname{tr}\left(H_{j} \rho_{\beta} H_{j}^{*}\right) \operatorname{tr}\left(V_{j} \rho_{\beta} V_{j}^{*}\right)\right) \operatorname{tr}\left(W_{j} \rho_{W} W_{j}^{*}\right) \leq \log \beta
$$


where equality holds if and only if for all $i, j$,

$$
\frac{1}{\beta} \operatorname{tr}\left(H_{j} \rho_{\beta} H_{j}^{*}\right) \operatorname{tr}\left(V_{j} \rho_{\beta} V_{j}^{*}\right)=\frac{\operatorname{tr}\left(W_{j} V_{i} \rho_{W} V_{i}^{*} W_{j}^{*}\right)}{\operatorname{tr}\left(V_{i} \rho_{W} V_{i}^{*}\right)}
$$

As we have discussed before, it is not clear that given any dynamics, we can obtain a measure such that we can reach the maximum value $\log \beta$. Considering particular cases, we can suppose, for instance, that the $V_{i}$ are unitary. In this way, we combine in a natural way a problem of classic thermodynamics, with an evolution which has a quantum character. In this particular setting, we have for each $i$ that $V_{i} V_{i}^{*}=V_{i}^{*} V_{i}=I$ and then the basic inequality becomes

$$
h_{V}(W)+\sum_{j=1}^{k} \operatorname{tr}\left(W_{j} \rho_{W} W_{j}^{*}\right) \log \operatorname{tr}\left(H_{j} \rho_{\beta} H_{j}^{*}\right) \leq \log \beta
$$

and equality holds if and only if for all $i, j$,

$$
\frac{1}{\beta} \operatorname{tr}\left(H_{j} \rho_{\beta} H_{j}^{*}\right)=\operatorname{tr}\left(W_{j} V_{i} \rho_{W} V_{i}^{*} W_{j}^{*}\right)
$$

We have the following:

Lemma 12 Given a QIFS with a unitary dynamics (i.e., $V_{i}$ is unitary for each $i)$, there are $\hat{W}_{i}$ which maximize (110), i.e., such that

$$
h_{V}(\hat{W})+\sum_{j=1}^{k} \operatorname{tr}\left(\hat{W}_{j} \rho_{\hat{W}} \hat{W}_{j}^{*}\right) \log \operatorname{tr}\left(H_{j} \rho_{\beta} H_{j}^{*}\right)=\log \beta
$$

The above lemma also holds for the basic inequality in coordinates, given by (89). Also, it is immediate to obtain a similar version of the above lemma for any QIFS such that the $V_{i}$ are multiples of the identity, and also for QIFS such that $\rho_{W}$ fixes each branch of the QIFS, that is, satisfying

$$
\frac{V_{i} \rho_{W} V_{i}^{*}}{\operatorname{tr}\left(V_{i} \rho_{W} V_{i}^{*}\right)}=\rho_{W}
$$

\section{References}

1. Baraviera, A., Lardizabal, C. F., Lopes, A. O., Terra Cunha, M. A Thermodynamic Formalism for density matrices in Quantum Information. Applied Mathematics Research Express, Vol. 2010, No.1, pp. 63-118. 
2. Baraviera, A., Lardizabal, C. F., Lopes, A. O., Terra Cunha, M. Quantum Stochastic Processes, Quantum Iterated Function Systems and Entropy. To appear on São Paulo Journal of Mathematical Sciences (2010).

3. Benatti, F. Dynamics, Information and Complexity in Quantum Systems. Springer, 2009.

4. Benenti, G., Casati, G. Strini, G. Principles of Quantum Computation and Information, Vol I and II, World scientific, 2007

5. Bengtsson, I., Życzkowski, K. Geometry of Quantum States. Cambridge University Press, 2006.

6. Busch, P., Ruch, E. The measure cone: irreversibility as a geometrical phenomenon, Int. J. Quantum Chemistry. 41, 163-185, 1992.

7. Castro, G., Lopes, A. O. KMS States, Entropy and a Variational Principle for Pressure, to appear in Real Analysis Exchange (2009).

8. Gardiner. C. W., Zoller, P. Quantum Noise, Springer Verlag, 2004.

9. Gray, R. M. Entropy and information theory. Springer-Verlag, New York, 1990.

10. de Gosson, M. Symplectic geometry and quantum mechanics. Birkhauser, 2006.

11. Gustafson, S., Sigal, I. Mathematical Concepts of Quantum Mechanics. SpringerVerlag, 2003.

12. Hastings, M. B. A counterexample to additivity of minimum output entropy. arXiv:0809.3972 v3 [quant-ph], 2008.

13. Hayashi, M. Capacity with energy constraint in coherent state channel. arXiv:0904.0307v1 [quant-ph], 2009.

14. Hayashi, M., Nagaoka, H. General formulas for capacity of classical-quantum channels. IEEE Transactions on Information Theory, 7, v. 49, 2003.

15. Jordan, T. Affine maps of density matrices. Physical Review A, 71, 034101, 2005.

16. Lardizabal, C. F. Processos Estocásticos Quânticos, Tese de doutorado Prog. Posgrad. Mat. UFRGS - to appear (2010).

17. Lasota, A., Mackey, M. Chaos, fractals and noise. Springer-Verlag, New York, 1994.

18. Lopes, A. O., Oliveira, E. Entropy and variational principles for holonomic probabilities of IFS. Discrete and Continuous Dynamical Systems Vol. 23, N, 3, Series A. 937-955, 2009.

19. Lopes, A. O. Entropy and Large Deviation. NonLinearity Vol. 3, N. 2, 527-546, 1990.

20. Lopes, A. O. An analogy of the charge distribution on Julia sets with the Brownian motion. J. Math. Phys. 30 (9), 1989.

21. Lopes, A. 0., Craizer, M. The capacity-cost function of a hard-constrained channel. Int. Journal of Appl. Math. Vol 2, N 10 pp 1165-1180 (2000).

22. Lozinski, A., Życzkowski, K., Słomczyński, W. Quantum iterated function systems, Physical Review E, Volume 68, 04610, 2003.

23. Mañé, R. Ergodic Theory, Springer Verlag, 1986.

24. Nielsen, M., Chuang, I. Quantum computation and quantum information. Cambridge University Press, 2000.

25. Parry, W., Pollicott, M. Zeta Functions and the Periodic Orbit Structure of Hyperbolic Dynamics. Société Mathématique de France. 187-188, Astérisque, 1990.

26. Rieffel, E., Polak, W. An Introduction to Quantum Computing for Non-Physicists, ACM Computing Surveys, Vol. 32, Issue: 3, p. 300-335, 2000.

27. Shor, P. W. Equivalence of additivity question in quantum information theory. Comm. Math. Phys. 246, 453-472 (2004).

28. Słomczyński, W., Życzkowski, K. Quantum Chaos: an entropy approach. J. Math. Physics, 32 (1), 1994, p. 5674-5700. 
29. Słomczyński, W., Dynamical Entropy, Markov Operators and Iterated Function Systems. Jagiellonian University Press, 2003.

30. Spitzer, F. A Variational characterization of finite Markov chains. The Annals of Mathematical Statistics. (43): N.1 303-307, 1972.

31. Srinivas, M. D. Foundations of a quantum probability theory Journal of Math. Phys., Vol. 16, No. 8, 1975.

32. Winkler, G., Choquet Order and Simplices. Lecture notes in Mathematics 1145. Springer-Verlag, Berlin, 1985. 